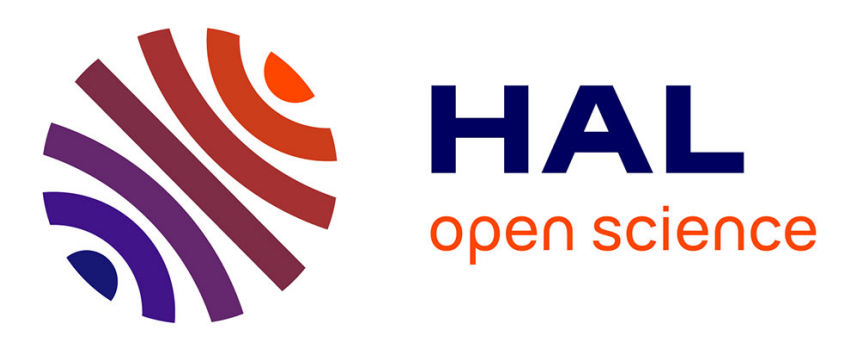

\title{
Parole, corporéité, individu et société : l'embodiment entre le représentationnalisme et la cognition incarnée, distribuée, biosémiotique et enactive dans les linguistiques cognitives
}

Didier Bottineau

\section{To cite this version:}

Didier Bottineau. Parole, corporéité, individu et société: l'embodiment entre le représentationnalisme et la cognition incarnée, distribuée, biosémiotique et enactive dans les linguistiques cognitives. Intellectica - La revue de l'Association pour la Recherche sur les sciences de la Cognition (ARCo), 2011, 56, pp.187-220. halshs-00656024

\section{HAL Id: halshs-00656024 \\ https://shs.hal.science/halshs-00656024}

Submitted on 3 Jan 2012

HAL is a multi-disciplinary open access archive for the deposit and dissemination of scientific research documents, whether they are published or not. The documents may come from teaching and research institutions in France or abroad, or from public or private research centers.
L'archive ouverte pluridisciplinaire HAL, est destinée au dépôt et à la diffusion de documents scientifiques de niveau recherche, publiés ou non, émanant des établissements d'enseignement et de recherche français ou étrangers, des laboratoires publics ou privés. 
Didier Bottineau - CNRS, MoDyCo, Université Paris Ouest

\title{
Parole, corporéité, individu et société : l'embodiment entre le représentationnalisme et la cognition incarnée, distribuée, biosémiotique et enactive dans les linguistiques cognitives
}

\begin{abstract}
Résumé
En linguistique cognitive, le terme de corporéité (embodiment) désigne la conceptualisation du rapport incarné du sujet au monde par l'engagement moteur et sensoriel multimodal, et les traces que laissent ces représentations dans les formalismes langagiers (organisation du lexique, constructions). Dans la présente étude, on montre que l'embodiment en LC n'a pas été pris en compte dans la définition du signifiant et dans la caractérisation de l'acte de parole en tant qu'expérience vivante (languaging). Pour rendre compte de ce fait, on le resitue dans le contexte historique de l'émergence de la LC au sein de la révolution cognitiviste, son positionnement ambigu par rapport au générativisme, et le rendez-vous manqué avec des modèles cognitifs pré-cognitivistes comme la psychomécanique du langage. Et pour le dépasser, on présente un courant de pensée actuel qui s'achemine vers une refondation de la problématique de la parole vécue à l'interface de la cognition incarnée par l'individu et de la cognition sociale intersubjectivement distribuée. Ce croisement permet d'échapper à une approche représentationnaliste de type encodagiste, reconsidère la parole comme un processus et une modalité de l'action, précise la nature de la dynamique, et précise les modalités d'application du programme de recherche en redéfinissant dans cette perspective les catégories analytiques traditionnelles (lexique, morphosyntaxe, prosodie) dans leur variation typologique au sein des langues naturelles.
\end{abstract}

\begin{abstract}
Languaging, corporality, the individual and society: embodiment between representationalism and embodied, distributed, biosemiotic and enactive cognition in cognitive linguistics

In cognitive linguistics, the word embodiment refers to the conceptualization of the subject's embodied relation to the world through her motoric and multimodal sensorial involvement, along with the traces left by these representations within natural linguistic formalisms (the lexicon and constructions). In this paper, it is shown that in LC, embodiment has not been integrated into the definition of the signifier and in the characterization of speech in terms of living experience, or languaging. To account for this fact, it is to be resituated in the historical context of the emergence of $L C$ within the cognitivist revolution paradigm; its ambiguous positioning in relation to generativism is to clarified, along with its connection with pre-cognitivist models such as the psychomechanics of language. And in order to go beyond those limitations, the paper presents a currently growing paradigm which paves the way for a refounding the problematic of languaging at the interface of the subject's embodied cognition on the one side and intersubjectively distributed social cognition on the other. This correlation makes it possible to evade a representationalistic approach based on encodingism. It reconsiders speech as a process and a modality of action, defines the content of this dynamic, and specifies how this research programme is to be applied by redefining in this perspective the traditional analytical categories (lexicon, morphosyntax, prosody) in their typological variations among natural languages.
\end{abstract}




\section{Mots-clés}

Linguistique cognitive, générativisme, psychomécanique du langage, parole, languaging (« parlance »), embodiment (corporéité), représentation, enaction, cognition distribuée, biosemiosis

\section{Key words}

Cognitive linguistics, generativism, psychomechanics of language, speech, languaging, embodiment, representation, enaction, distributed cognition, biosemiosis

Guignard (2008) souligne a plusieurs reprises l'ancrage représentationnel de la corporéité en linguistique cognitive. Cette position se cristallise par quelques qualifications transparentes que l'on trouvera synthétisées dans le présent volume: la LC est un «nativisme représentationnel », un «réalisme incarné », un « internalisme qui ne rompt pas avec la tradition qu'elle décrie par ailleurs ». Cette tradition se caractérise, en effet, notamment par un binarisme radical corps / esprit (comme le détaille Keller, 2006), l'étonnante imprécision de la définition de la notion d'embodiment qu'elle reconnait (particulièrement chez Lakoff et Johnson 1999 comme le fait remarquer Zlatev 2008 ; cette définition sera précisée, entre autres, par Wilson 2002 et Ziemke 2003); et le «mentalisme exclusif» qui fait de «l'expériencialisme » représentationnaliste de la LC un «modèle idéaliste dont la seule incarnation est « déjà » conceptuelle »(Guignard, ibid.).

La triple articulation embodiment, constructique et catégories, et la valeur symbolique des structures syntaxiques dans un modèle voulu non modulariste, soulève la question de la place de la corporéité dans la théorie du langage et des langues proposée par ce paradigme. Le fond du problème est simple: en linguistique cognitive, le terme de corporéité (embodiment) désigne essentiellement la conceptualisation du rapport incarné du sujet au monde par l'engagement moteur et sensoriel multimodal, et les traces que laissent ces représentations dans les formalismes langagiers (organisation du lexique, constructions). En dehors de certaines directions théoriques particulières, la dimension incarnée des actes de signification eux-même ne semble pas jouer un rôle central.

Dans les lignes qui suivent, on explore la question de la corporéité de l'expérience de la parole pour le sujet dans l'environnement matériel-symbolique, dont le rapport intersubjectif. La première section précise en quoi on peut considérer le signifiant comme désincarné en LC. La deuxième explique cette caractéristique par le positionnement ambigu de la LC : actrice de la révolution cognitiviste comme la grammaire générative, mais fondée ultérieurement en réaction à l'évolution du paradigme générativiste lui-même. La troisième section expose l'articulation du traitement de la corporéité conceptuelle en LC par rapport à certains éléments centraux de la psychomécanique du langage, fondée par «l'aïeul tutélaire de la linguistique cognitive à la française » selon Rastier 1993, et vise à expliciter a posteriori un débat qui n'a pas eu lieu à l'époque. La quatrième et dernière section présente des développements actuels qui visent à sortir des apories laissées en suspens et expose les propositions issues de théories liées à l'enaction, la biosemiosis, la cognition distribuée. Il s'agit de considérer la parlance (languaging) comme un processus dynamique distribué sur les corps et l'environnement matériel dans sa dimension socio-sémiotique, de se demander comment un individu donné se développe et apprend en se faisant recruter par sa participation corporelle au processus collectifs, et de s'interroger sur la nature des effets produits, sur le groupe comme sur le sujet, par cette dynamique biosémiotique. En tout état de cause, il sera nécessaire de renoncer à l'approche encodagiste et radicalement symbolique, de repenser la parlance comme une 
activité processuelle fondatrice d'une forme particulière du sens, et de tirer les conséquences de l'inévitable inscription corporelle des processus sémantiques et mentaux.

\section{En linguistique cognitive (LC), le signifiant est désincarné, seul est incarné le signifié}

Le programme théorique de la LC ne lui appartient pas en propre à l'origine : l'objectif de Johnson 1987, dans l'ouvrage au titre explicite The Body in the Mind: The Bodily Basis of Meaning, Imagination, and Reason, était de montrer comment une théorie générale du sens et du raisonnement devait se fonder sur la prise en compte de l'expérience «corporelle » (bodily), à savoir sensorimotrice. L'ouvrage fondateur de Lakoff et Johnson 1980, Metaphors we live by, plutôt qu'une théorie linguistique, recherche dans les faits langagiers les indices empiriques de la corporéité du sens en général, laquelle n'est pas propre au fait langagier. Il est donc tout à fait normal que ces auteurs se soient intéressés à l'ancrage corporel des structures conceptuelles signifiées tout en se détournant de celui des actes de signification qui en sont les symptômes.

La LC considère les structures langagières comme le reflet ou l'encodage de structures conceptuelles sous-jacentes. Elle se fixe un double objectif: (i) la modélisation des représentations internes par les catégories, les schèmes-images et scenarii mentaux; (ii) l'inventaire des réalisations lexicales et constructionnelles qui en réalisent l'affichage à la surface empirique des manifestations comportementales. Dans cette architecture, les propriétés descriptives des langues, telles que les traces des processus de catégorisation par la polysémie lexicale ou la sémantique des constructions, sont asservies à la dynamique conceptuelle posée en amont: la démarche est de type descendant (top-down), onomasiologique du point de vue du linguiste. Les topologies cinématiques postulées pour le niveau conceptuel sont élaborées à partir de travaux menés sur la perception, l'action, l'attention, l'émotion et la mémoire empruntés au domaine de la psychologie, et plus particulièrement la psychologie elle-même cognitive, centrée sur l'étude de la relation de l'individu au «monde». De ce fait, les éléments candidats à la conceptualisation sont envisagés comme la partie égocentrée de l'expérience vécue : l'organisation conceptuelle de l'espace, du temps, de la relation topologie / action / géographie, les processus de catégorisation, les projections métaphoriques et la reconnaissance ou constitution d'analogies. Ceci est en soi problématique, dans la mesure où à aucun moment de son existence le sujet n'est exposé à l'expérience de «l'espace», le «temps», les «situations » (états de l'environnement), les « objets », les «évènements », « autrui » (comme partenaire et comme acteur), ni même à l'expérience réflexive de ses propres actions dans leur dimension psychogénétique (Bégin 1998) et épiphylogénétique (Stiegler 2004), hors du commerce des relation avec autrui, qu'elles soient verbalisées ou non : un acte individuel de catégorisation est-il seulement envisageable ? Les catégories et les schèmes, même autres que langagiers, ne sont-ils pas voués à constituer des processus socio-culturels constitutifs des sujets du fait de l'ancrage interactionnel et des conditions interactives de leur expérienciation ${ }^{1}$ ? Admettons-le pour le moment, mais avec un coût théorique important - l'obligation de croire, provisoirement, en un monde matériel objectif potentiellement porteur d'une sémantique primitive universelle, schématisable empiriquement par le sujet, modélisable par les théories,

\footnotetext{
${ }^{1}$ Rastier 2006 : «Le langage est un milieu et non une simple faculté ». (...) «Ni interne ni externe, la langue est ainsi un lieu du couplage entre l'individu et son environnement, parce que les signifiants sont externes (bien que reconstruits dans la perception) et les signifiés internes (bien que construits à partir d'une doxa externe). Comme le langage fait partie du milieu dans lequel nous agissons, c'est dans des pratiques diversifiées, dont témoignent les discours et les genres, que nous nous lions à notre environnement. Mais il est aussi peuplé de choses absentes, et dans l'expérience de l'altérité, du passé, de l'étranger, la culturalisation de l'enfant a lieu non moins sinon plus que dans l'expression d'une expérience individuelle limitée au hic et nunc ».
} 
et responsable de la relative convergence des « représentations » que l'on croit observer dans les différentes langues.

Sous cet angle, la LC parvient à faire une certaine place à la corporéité : les schèmes-images sont construits, extrapolés et abstraits à partir d'occurrences expériencielles ancrées dans l'intéraction sensorimotrice du corps au monde ; aussi les représentations conservent-elles une trace de ce rapport. Selon les approches, ce rapport sera essentiellement visuel (la grammaire cognitive de Langacker); ou plus multimodal (la dynamique des forces de Talmy, qui suppose la prise en compte du champ gravitationnel, lui-même construit par recoupement d'indicateurs sensoriels multimodaux incluant la vue, l'ouïe pour l'équilibre, le toucher pour la résistance des supports au contact et au mouvement); il peut prendre en compte le rôle de la motricité, comme l'attribution des propriétés «haut» et «bas» par projection de l'expérience motrice requise pour atteindre les positions concernées relativement au point de vue adopté, et la participation adjuvante ou opposante du champ gravitationnel relativement à ces mouvements conceptuels métaphoriques - on monte ou descend à Paris en fonction de (i) la hiérarchisation culturelle des loci considérés, (ii) le choix du locus comme point de vue, et (iii) le statut culturel du déplacement, reconnu comme ordinaire ou exceptionnel et requérant un effort. Une préposition comme sous traite conjointement les dimensions visuo-spatiale et tacto-gravitationnelle: le chat est sous la couverture $=$ (i) hors du champ visuel de l'observateur et (ii) en situation d'expérience, de ressentir l'effet du poids de l'objet (confinement protecteur et chaleur); mais dans les locutions prépositionnelles figées, ces facettes spécialisées sont sélectionnées de manière à s'exclure mutuellement (Bottineau 2008b et 2010f) : la situation est sous contrôle (= sous l'influence d'une force humaine analogue à celle du champ gravitationnel) vs sous couleur / prétexte de (couleur et prétexte $=$ des masques ou caches visuels, comme la couverture posée sur le chat). De manière générale, les locutions prépositionnelles sélectionnent un aspect de la matrice d'impressions multimodales concentrées par «l'invariant » de la préposition (ou par «l'air de famille » qui lie les emplois selon Vandeloise 1986), si bien que la comparaison de ses emplois dans et hors figement livre un cadre méthodologique régulé permettant de circonscrire les composantes de la corporéité conceptuelle qu'on lui prête.

Cependant, « l'embodiment » dont il est ici question est celui de la « cognition » en temps que processus de conceptualisation porté par l'expérience motrice et sensorielle individuelle de la dimension physique du monde matériel, y compris le corps propre de l'expérient dans le meilleur des cas. Il ne s'agit pas de l'expérience des actes de parole eux-même, et à aucun moment il n'est question en LC d'une théorie du signifiant, à quel niveau de segmentation que ce soit (morphème, lexique, syntagmes, propositions, discours, dialogue) qui reconstruise l'expérience sensorimotrice de la parole comme processus biosémiotique (Hoffmeyer 2008) et interactionnel contextualisé dans le tissu d'un dynamisme constitutif d'un réseau ou d'un processus social (Bang \& Døør 2007, De Jaegher \& Di Paolo 2007, Bottineau 2010c). Une partie de la LC s'appuie sur l'usage (est usage-based, s'opposant au point de vue encore défendu par Newmeyer 2003 : " Grammar is grammar, and usage is usage »), mais le point de vue phénoménologique adopté sur l'usage est externe et quantitatif, se fixant des objectifs ne dépassant guère l'observation des faits d'usage et l'analyse statistique de la distribution des manifestations constatées dans les classes d'interactions accessibles à l'observation. La LC, en tant que linguistique, n'est pas experience-based, elle ne se fixe pas pour objectif d'étudier l'importance des conditions matérielles d'expérienciation de la motricité et sensorialité de la verbalisation même du côté des signifiants langagiers, que l'on envisage la chose du point de vue du producteur moteur apparent, le «sujet parlant», ou du consommateur apparent de signal acoustique, le «sujet écoutant». Il peut sembler surprenant que la revendication de l'embodiment soit restée lettre morte pour tout ce qui intéresse la manifestation empirique du 
fait de parole dans sa double dimension motrice et sensorielle. En fait, la chose découle naturellement des présupposés internalistes représentationnels propres au cognitivisme : dès lors que la binarité forme / sens, grammaire / conceptualisation, est considérée comme allant de soi, la «forme »n'a plus à être interrogée dans sa forme même ; le « sens » est formalisé dans les termes d'une discipline d'emprunt, la psychologie, sous la forme de la Gestalt notamment; et le rapport se gère par la recherche des correspondances.

\section{La LC révolutionnaire}

Comme le démontre JBG, on peut qualifier le programme de la LC de réalisme incarné conduisant à un internalisme nativiste, parfois explicitement revendiqué, comme la lingua mentalis de Wierzbicka ${ }^{2}$. Sous cet angle, la LC radicalise, sans le reproduire pour autant, un penchant internaliste que l'on observait déjà chez le linguiste français Gustave Guillaume (1883-1960), penchant que radicalise également Bernard Pottier bien avant l'avènement de la LC ; et dans le même temps, elle rejette l'orientation générativiste, qui avait paradoxalement été préparée elle aussi par la psychomécanique du langage. Explicitons ceci point par point.

\section{- L'intelligence artificielle computationnelle, mimesis de l'intelligence naturelle?}

A la fin du dix-neuvième siècle, l'introspectionnisme en psychologie menace de s'effondrer sous le poids de la prolifération de modèles non convergents rendue possible par le manque de protocole méthodologique et l'infalsifiabilité expérimentale des hypothèses (Gardner 1985). Avant même que ce dénouement ne survienne, l'introspectionnisme se retrouve marginalisé par l'avènement du behaviorisme et la généralisation du schème comportemental stimulus / réponse, généralisable à l'ensemble des comportements humains observables et testables : le mentalisme est ignoré ou réduit à des déterminismes tangibles. Comme toute révolution, le behaviorisme est un apport positif par la volonté de résoudre le type de difficultés sur lesquelles il a concentré sa dénonciation, et négatif par les excès engendrés par son réductionnisme hégémonique. Il appelait donc son propre dépassement, lequel est opportunément rendu possible par la cybernétique et l'émergence de (ce qui s'auto-baptise) l'intelligence artificielle et des sciences cognitives : avec le test de Turing, on en vient à penser qu'il est possible d'imaginer des «machines » dont les réponses peuvent passer pour être d'origine humaine. Le sujet humain qui reçoit la réponse la reconnaît conforme à ce qu'un humain produirait dans la même position, et ne peut s'empêcher d'attribuer à la machine les prérequis cognitifs qu'un humain mettrait en œuvre pour réaliser la même tâche : le test joue sur une illusion cognitique. Avec l'avènement de l'ordinateur, ces parcours se matérialisent $^{3}$, les computations deviennent des métaphores de la cognition incarnée, et le rapport hardware / software fournit un modèle, certes extrêment rudimentaire, mais tout de même un modèle, du rapport esprit / corps chez l'humain en général, et en particulier de son sous-ensemble local, le rapport intelligence (naturelle) / cerveau.

\footnotetext{
${ }^{2}$ Pour une critique récente de la circularité méthodologique des «métalangages sémantiques naturels », cf. Wawrzyniak 2010.

${ }^{3}$ Ceci concerne aussi les robots, même sans logiciel ni mémoire. Par exemple, on peut aisément concevoir une salamandre-robot amphibie, dont les roues motrices sont activées par le poids du chassis sur les amortisseurs utilisés comme interrupteurs, alors que l'hélice de propulsion est activée par le poids du chassis sur les flotteurs latéraux en cas de flottaison, également en fonction d'interrupteur. Résultat : «l'animal » se met à rouler seul quand on le dépose au sol, et commute seul d'un mode de locomotion à l'autre en fonction du terrain rencontré sur une plage de sable (résistance du sol sous les roues vs résistance de l'eau sous les flotteurs) sans intervension extérieure, ni détection, ni processus calculatoire ou décisionnel, donnant l'impression d'un être vivant qui perçoit un milieu qu'il connaît bien et adapte son action aux circonstances. Pour un observateur non averti, il est difficile de ne pas attribuer au robot pragmatique des qualités sensorimotrices et cognitives propres au vivant.
} 
Or - la chose est triviale mais constitue le départ de l'analyse - il se trouve que l'ordinateur n'a pas de corps ; il opère des calculs, langagiers ou autres, qu'il « réussit » (du point de vue humain) en dépit de l'absence de rapport expérienciel incarné à l'espace environnant par la sensorimotricité : l'ordinateur désincarné opère par manipulation computationnelle de symboles abstraits, dénués de corrélats sémantiques, indexicaux, occurrentiels et situés; pourtant, il fonctionne correctement. Cette analyse est évidemment fausse dans la mesure où l'ordinateur, loin d'être un agent autonome, est une machine créée par l'humain et qui ne fonctionne que dans le cadre des rapports processuels entretenus avec l'humain, ne serait-ce que par sa mise sous tension, le lancement d'un logiciel et d'une application, la demande d'exécution d'une tâche - ce qui en fait le prolongement artificiel culturalisant d'une intelligence naturelle elle-même déjà largement culturalisée par les processus sociaux où elle s'inscrit, à commencer par le langage.

L'idée selon laquelle l'IA serait nécessairement une métaphore pertinente de l'IN se base sur un double malentendu : l'un d'ordre sémiotique, celui de croire que l'indétermination de la source humaine ou robotique de l'information garantit la légitimité de l'amalgame des procédures exécutoires mises en œuvre par l'un et l'autre; l'autre a trait à la construction phénoménologique : on ne peut considérer la machine comme un agent autonome supplétif à l'humain alors qu'on a affaire au minimum à un prolongement complétif, à l'origine du paradigme de la cognition étendue par la technologie (Dror \& Harnad 2008) ${ }^{4}$.

Sous cette formulation lapidaire, le glissement peut sembler surprenant, mais pour en comprendre la motivation, il faut se resituer dans le contexte de la fascination bien compréhensible pour la cybernétique, l'IA, l'informatique et la robotique d'une part, et d'autre part le rôle catalyseur du financement massif de ces programmes par des institutions publiques et privées qui y voyaient un intérêt majeur tant technologique (civil et militaire) que commercial et publicitaire (y compris le mécénat philanthropique), situation que l'on n'a jamais connue en Europe.

\section{- A l'origine du programme générativiste : la «pauvreté du stimulus »}

Dans la nébuleuse interdisciplinaire des sciences cognitives émergentes, Chomsky opère une projection radicale : en vertu de la «pauvreté du stimulus », l'humain ne dispose pas dans son expérience individuelle du rapport au monde des indicateurs interprétables nécessaires à la formation d'un savoir-faire génératif langagier stabilisé, la «compétence », susceptible de rendre possible la «performance », la production d'un nombre ouvert d'énoncés corrects et reconnus conforme à la régulation par l'intuition de grammaticalité inhérente à tout locuteurauditeur idéal. Par la "pauvreté du stimulus », et dans un contexte où le principal souci était de disqualifier les déterminismes matériels behavioriens d'origine externe au profit d'une internalisation du siège des processus mentaux, Chomsky a considéré que le corps et

\footnotetext{
${ }^{4}$ Pour être précis : si on conserve le clivage internalisme / externalisme, alors on analysera la machine comme une extension prosthétique des facultés humaines en fonction d'outil bilatéral, tourné à la fois vers l'action sur le monde (dont autrui) et l'action réflexive (sur soi-même), par exemple : le renoncement à l'orthographe grâce aux correcteurs automatiques, le renoncement au «sens de l'orientation » et à la lecture de cartes grâce au GPS, etc. Mais si on considère ces processus technologiques comme le milieu dans lequel l'humain se plaît à vivre et se fait évoluer par un jeu d'adaptation réciproque, alors il faut parler de cognition distribuée entre agents autopoïétiques (les humains vivants autonomes et en situation d'adaptation et d'évolution) et agents allopoḯtiques (l'ensemble de leurs créations hétéronomes et des processus qu'elles impriment dans l'environnement). La cognition est alors celle du milieu en tant que domaine conventionnel d'interactions (dans la terminologie de Maturana), et la reconnaissance «d'intelligences situées » par les observateurs humains réside en fait dans l'attribution de propriétés autopoiétiques aux agents rencontrés, vivants ou non. Le test de Turing consiste avant tout à stimuler chez l'observateur l'attribution d'un statut autopoiétique au processus constaté chez une machine, c'est-à-dire à attribuer le fait cognitif postulé à un agent spécifique plutôt qu'à un réseau relationnel dont l'observateur fait lui-même partie.
} 
l'expérience ne jouaient aucun rôle structurant dans le fait cognitif, lequel est désincarné, calculatoire, symbolique, autonome (fonctionnant sans référence à l'expérience du monde dont le rapport à autrui et à soi-même), et non acquérable par apprentissage, donc strictement intérieur et inévitablement inné. On n'insistera pas sur le potentiel quasiment créationniste d'une telle position si n'est pas résolue de manière satisfaisante l'incompatibilité entre innéisme cognitif et évolutionnisme darwinien des espèces, notamment dans leur organisation sociale et leur rapport à l'environnement.

Cette posture explique la grammaire universelle et le choix d'une unité de production déliée de la mémoire de l'expérience de la parole, la structure syntaxique qui sous-tend la phrase minimale $(\mathrm{P}=\mathrm{SN}+\mathrm{SV})$ : l'objet à produire est la phrase en tant qu'objet modélisé. Le schème syntaxique n'a pas en soi de réalité empirique; l'ensemble des phrases qui le manifestent n'est pas enregistré par la mémoire à long terme sous la forme de citations ou d'exemplaires épisodiques. La connaissance de la structure syntaxique ne peut pas être extraite de la pratique de l'expérience : le savoir-faire structural ne s'acquiert pas comme une catégorie, et il relève d'une compétence innée. La grammaire générative a connu plusieurs générations de formalismes, dont un, le programme minimaliste, qui relativise la pauvreté du stimulus: seuls les principes inhérents au langage sont universaux, mais les paramètres propres à chaque langue se spécifient dans le cadre de l'interaction à travers les rapports sociaux, expliquant simultanément pourquoi l'acquisition de la parole est inévitable chez tout enfant ne souffrant d'aucune pathologie physique ou mentale rédhibitoire mais exige l'inclusion de l'individu dans la dynamique des rapports sociaux verbalisés. Cet amendement sauve l'internalisme radical en expliquant comment son contenu s'ajuste par interaction avec des contraintes externes, mais il ne résout pas son incompatibilité avec l'évolutionnisme darwinien ou autre.

Dans ce système, les langues sont des ensembles architecturés de symboles non mimétiques, qui n'encodent ni l'expérience du monde, ni le processus calculatoire, lequel est à modéliser par un formalisme ad hoc: Chomsky en a proposé plusieurs, et d'autres ont suivi et approfondi la démarche. Le parallélisme méthodologique entre l'intelligence artificielle et l'intelligence naturelle pour le langage est clair : de même que l'ordinateur sans corps sensible procède par manipulation computationnelle de symboles abstraits internes déliés des choses du monde, l'intelligence humaine, coupée qu'elle est malgré son corps des choses du monde par la pauvreté du stimulus, est nécessairement équipée «en série » d'un logiciel calculatoire inné aux propriétés analogues à celui de l'IA. Le déplacement est non moins clair : de même que l'ordinateur n'est pas un agent autonome mais une prothèse épiphylogénétique de l'être humain socialisé et culturalisé par l'expérience totale des pratiques qui fédèrent les groupes et l'espèce, le corps sensible n'est pas confronté à un stimulus indigent, mais bien à une relation phénoménologique qui coordonne les émergences du «sujet» et de «l'objet» en voie de constitution par le processus de l'interaction (Dinis 2010). De même qu'on peut refuser à l'ordinateur le statut de prolongement du corps humain, on peut refuser au cerveau humain le statut d'organe vivant d'un corps vivant dans un monde matériel et en partie vivant. De même que la cognition est étendue techniquement, ou articulée sur un rapport liant l'esprit à la machine, elle est étendue biomécaniquement (Clark 2008), y compris par le langage (Steffensen 2009), ou distribuée sur le réseau relationnel (pour le langage, Cowley 2007 et 2009).

Ceci a trait en particulier à la question de la non-acquérabilité des connaissances et compétences par le truchement de l'expérience, qui concerne en parallèle l'ordinateur (qui dispose d'un programme), l'individu face au langage (qui constituerait un programme) et le développement biologique de la cellule et du corps (qui serait déterminé par un génome). En génétique, l'idée d'un génome comme programmation intégrale est largement remise en 
cause : les jambes humaines ne deviennent aptes à porter le corps par la marche que parce qu'elles se développent dans l'expérience du champ gravitationnel, avec l'épreuve d'une résistance environnementale qui appelle la croissance osseuse et musculaire adaptée. On sait que le développement des corps vivants végétaux et animaux hors gravitation, quand il est viable, est fortement orienté par la spécificité des caractéristiques environnementales locales et de l'adaptation divergente qu'elle induit; le génome enregistre un programme de développement adaptatif qui, pour la viabilisation de l'être, prévoit une dynamique ouverte de couplage à l'environnement selon un rapport de type dialogal, avec des répliques développementales, pragmatiques et gnosiques en réponse suscitée, mais non stimulée, par les répliques environnementales ambiantes ${ }^{5}$.

Comme le dit en quatre mots le journaliste musicologue Allemand J.E. Berendt (1922-2000), Die Welt is Klang « le monde est son » autant que gravitation, et dans le bruit ambiant il en est un qui immanquablement focalise l'attention du nouveau-né (et même du pas-encore-né dès le sixième mois de grossesse, voire bien avant selon le très controversé Tomatis) et procure toute satisfaction, soulagement et bien-être dans les situations de détresse physique (la faim) et psychique (l'angoisse) : la voix humaine, promesse de l'intervention réconfortante maternelle ou paternelle. La parole est l'un de ces paramètres environnementaux incontournables, au même titre que la gravitation, qui se présente au nouveau-né en fonction d'interlocuteur et d'épreuve adaptative, auxquels s'adaptera inévitablement son développement initiatique tant que cette orientation est ressentie comme génératrice de bien-être efficace pour la survie et enregistré comme désirable, donc intentionnalisable: de même que l'apprentissage de la marche lève l'obstacle gravitationnel de l'ancrage géographique et inaugure l'exploration initiatique du monde matériel, la parole lève l'obstacle de l'ancrage égotique de l'expérience et inaugure l'exploration initiatique de l'intersubjectivité au sein du groupe et de l'espèce (chose que Berendt formule cette fois Vom Hören der Welt: Das Ohr ist der Weg «De l'audition du monde: l'oreille est la voie »). La surdité peut perturber collatéralement l'apprentissage langagier des normes sociales de l'intellection interactive, l'insertion dans la dynamique collective, et le regard du groupe sur le devenir du sujet non recruté, tant par un jugement erroné sur les causes de la non- ou mé-participation que par des pratiques d'insertion inadaptées ou inexistantes (Poizat 1996). S'il est un « résidu inné » dans la parole, il faut sans doute le rechercher du côté du programme d'organisation biomécanique du corps lui-même : les neurones miroirs et leur capacité à coactiver des motricités conjointes sans apprentissage préalable et avant même que le système phonatoire ne soit opérationnel pour les produire, comme le suggèrent les travaux récents d'E. Dehaesne. Le langage n'est probablement pas inné, mais son apprentissage, à l'instar de celui de la marche, passe par le développement de synchronisations biomécaniques intersubjectives qui, elles, sont rendues inévitables par des faits de structure enregistrés par l'ADN, à savoir, la mémoire continue de l'espèce vivant une expérience ininterrompue - l'ADN étant une partie du corps comme tout le reste (et non un code gravé sur un support pour le coup inconcevable). En somme, si le développement du «corps »du sujet est muni d'un dispositif d'orientation par apprentissage

\footnotetext{
${ }^{5}$ Un exemple spectaculaire de ce mécanisme est fourni par une découverte récente : suite à l'éruption du Mont Saint Helens, une variété locale de salamandre, privée de son alimentation terrestre par les arbres abattus, a survécu en se contentant de sa nourriture aquatique. S'abstenant de ses sorties d'eau, elle a "pris l'habitude » d'accéder à la matûrité reproductive en restant à l'état de larve, sans subir la métamorphose qui doit la conduire à l'état d'animal adulte. Un changement aussi radical en un temps aussi bref ne peut s'expliquer en termes de reprogrammation génétique «fermée » et précipitée par l'adaptation à une catastrophe environnementale. En revanche, si le rôle du génôme « ouvert» de cet amphibien consiste précisément à orienter le développement (dont la métamorphose éventuelle) en fonction des sollicitations fournies par l'environnement effectivement fréquenté, alors on comprend qu'une désertion soudaine de la zone terrestre se traduise aussi rapidement par le non-développement de la métamorphose qui en permet l'accès régulier.
} 
essentiellement fondé sur son intégration aux comportements sociaux par le biais de synchronisations biomécaniques intersubjectives, et si les principaux comportements sociaux en question sont la parole et la bipédie, alors l'apprentissage des deux est mécaniquement déterminée sans que l'on doive invoquer une compétence préalable ou un module computationnel monté en série, «inné ». L'humain, comme toute espèce vivante, serait naturellement programmé pour se culturaliser au contact des siens, et ceci ne fonctionne que si le «stimulus » est disponible (vs l'enfant sauvage), pleinement pris en compte et accepté En ce sens, le langage humain n'est pas plus «spécial » que toute autre originalité observée chez toute autre espèce, comme l'écholocation par clics chez les dauphins.

\section{- La réponse de la LC : le corps est dans la tête, mais comment y entre-t-il ?}

La LC s'est donc inscrite en faux contre l'innéisme internaliste modulariste et le computationnalisme symbolique de type chomskyen. L'argument central est que l'organisation du lexique et de la constructique dans les langues naturelles témoigne du rôle structurant de l'expérience motrice et sensorielle multimodale dans la structuration des représentations conceptuelles que les formes langagières encodent. Ce faisant, la LC a disjoint le côté $\mathrm{L}$ et le côté $\mathrm{C}$ : les actes de conceptualisation dans leur dimension non verbale sont inspirés par l'expérience sensorimotrice, et, en ce sens, «incarnés »; en revanche, les actes de parole, d'expression des images-schémas si l'on veut, ne sont pas envisagés dans leur dimension sensori-motrice du côté du signifiant, et sont à ce titre « désincarnés ». De plus, la question de savoir si la corporéité des représentations mentalisées résulte lui-même d'un processus d'apprentissage par l'expérience ou d'une sorte de module ontologique inné est en elle-même problématique en LC puisque l'on trouve dans la nébuleuse théorique des éléments qui tirent dans la direction de l'innéisme, comme les ISM, et d'autres dans celle de l'expériencialisme, comme la question des catégories.

La LC se présente comme un appariement $\mathrm{L}+\mathrm{C}$ hétérogène, une linguistique désincarnée de la cognition incarnée (d'une mentalisation pure de la corporéité), un inventaire des formes (envisagées sous un angle «désincarnant») qui encodent des représentations miexpériencielles, mi-innées, et formalisables. Ceci pose un problème de divergence phénoménologique : d'un côté, la LC s'intéresse à la corporéité de la sémantique lexicale et constructique; de l'autre, elle ignore la dimension biomécanique des signifiants et, par là même, leur potentiel biosémiotique propre, socialisé par l'interaction. Pourtant, cette dimension est de nature à requérir une révision profonde du contenu des actes de présentation conceptuelle vectorisés par la parole. Si la LC constitue un apport bénéfique en insistant sur la corporéité du sens des formes, elle néglige la corporéité des formes mêmes et de leur participation à la génération des effets de sens. Comme le souligne JBG, le bilan de la LC sera donc riche mais ambigu, avec de réels succès dans l'inventaire de propriétés linguistiques explicables par le «rapport du sujet au monde » (comme la spatialité de la conceptualisation du temps, ou le traitement des énoncés de mouvement par la distinction typologique entre verb-framed et satellite-framed languages chez Talmy), mais une bien réelle fragilité des résultats compte-tenu du silence réservé à la participation de l'expérience des actes verbaux

\footnotetext{
${ }^{6}$ Dans certains cas de dysphasie infantile, les déficiences observées s'expliquent non pas par des malformations ou traumatismes d'origine physique (lésions suite à accident) ou chimiques (traitement médicamenteux nocifs), mais par un rejet intuitif de la parole par l'enfant confronté à un milieu adulte environnant perçu comme dangereux, hostile, potentiellement traumatique pour cause de violence verbale, psychologique ou physique : l'enfant qui redoute de s'insérer dans le jeu des rapports sociaux redoutés, loin de désirer s'y intégrer par une participation adéquate et normée, pourra se protéger en produisant intentionnellement, si ce n'est délibérément, des formes verbales anormales, protectrices du fait de mener à l'exclusion. En pareil cas, le dysfonctionnement relève de la cognition distribuée sur le réseau intersubjectif, et il ne doit pas être attribué exclusivement à l'agent qui en manifeste et centralise les sympômes langagiers ; une remédiation inadaptée, fondée sur un diagnostic erroné, est susceptible d'aggraver la situation.
} 
dans les processus de conceptualisation en dépit de l'embodiment revendiqué, et plus généralement, une cécité vis-à-vis de la dimension sociale du rapport au monde même hors langage. Pour la LC, le corps agissant mais non-parlant est dans le cerveau, soit dès l'origine (innéisme), soit par expérience autre que de la parlance; le rôle de la parlance en tant qu'expérience corporelle constitutive de l'intériorisation en général n'est pas un thème central, ni du reste celui de l'existence d'une représentation des formats de l'interaction (comme on le trouve chez Douay 2000 sous la forme de l'appareil formel de l'interlocution). Ce n'est pas pour rien que l'embodiment n'est pas défini clairement : le faire revenait à mettre en lumière la divergence interne entre les traitements de l'incarnation du côté L et du côté $\mathrm{C}$.

Remarquons que la même ambivalence concerne la méthodologie. La LC emprunte ses outils conceptuels et terminologiques à la psychologie des «esquisses » ou des «silhouettes » (Gestalt), mais les modèles conceptuels qu'elle propose, comme les images-schémas, les espaces mentaux, les fenêtrages attentionnels, les intégrations conceptuelles, sont bel et bien interprétés directement à partir de l'observation des formes verbales collectées et «scrutées » par le linguiste sans passer par l'intermédiaire d'un protocole psycholinguistique en première instance (a posteriori, oui), ce qui revient virtuellement à pratiquer un introspectionnisme formalisateur qui ne dit pas son nom, avec divers problèmes méthodologique pour la validation (Fortis 1996). La LC n'est pas la seule à s'exposer au risque de cette critique, on la retrouve tout autant dans la psychomécanique du langage de Gustave Guillaume, la sémantique générale de Pottier, la théorie des opérations énonciatives de Culioli, la grammaire métaopérationnelle ou théorie des phases d'Adamczewski et bien d'autres - autant de modélisations et/ou formalisations d'architectures conceptuelles / représentationnelles / procédurales fondées sur l'interprétation des formes en contexte, éventuellement avec des corpus plus ou moins étendus (mais avec le risque, en ce cas, de dénaturer ou occulter le contexte et décontextualiser ou désincarner le processus introspectif, qui se retrouve vidé de son contenu).

\section{La LC et la PM (psychomécanique du langage)}

Pour comprendre le «projet total » de la LC, il est indispensable de le confronter à celui de la PM. Les fondateurs de la LC ignorent tout des développements «précognitifs » européens de la période qui précède (Rastier 1993, Fuchs 2004, Valette 2003, 2004 et 2006, Bottineau 2010c), et leur rejet du modularisme les amène à occuper, sans le savoir, des positions théoriques qui s'articulent précisément avec ces développements antérieurs, lesquels ne sont "précognitifs » que parce que le hasard de l'histoire de la pensée et de la terminologie linguistique a fait échoir la revendication de la thématique à la communauté disciplinaire américaine des années soixante-dix et suivantes.

Il se trouve que Guillaume avait, avant Chomsky, un projet à divers égards comparables à celui du fondateur du générativisme : rendre compte des conditions mentales nécessaires au savoir-faire langagier compris comme la production d'énoncés conformes à des modèles de génération et non préalablement rencontrés dans l'expérience sensible mémorisable, donc non reproductibles par répétition. Mais si Guillaume soulève une question analogue à celle de Chomsky, sa réponse part dans une direction diamétralement opposée. Avant Chomsky, Guillaume avait équipé le «psychisme humain »d'une sorte de logiciel, la «mécanique intuitionnelle», formalisée par le «tenseur binaire radical». L'approche est résolument internaliste, mais nullement innéiste ; au contraire, elle se veut naturaliste : l'idée de base est que le sujet confronté à l'environnement («l'homme dans l'univers») produit une figuration représentationnelle intériorisée de cet univers ( «l'univers dans l'homme ») sous une forme générale, théorique, et abstraite; mais que le sujet conserve en mémoire un souvenir 
représentatif de l'expérience de l'état primitif de la relation : ces deux états de l'accès à la connaissance du monde, «homme dans l'univers » et « univers dans l'homme », s'organisent en un schème dynamique à deux phases selon lequel l'accès au second état transite par la restauration du premier. Il s'ensuit un schème mental de type « universel - singulier universel » nommé tenseur binaire radical, psychologiquement matériel, théoriquement mesurable dans son exécution (le «temps opératif»). Selon Guillaume (Prolégomènes), ce schème abstrait, d'origine naturelle, émanant de la confrontation individuelle du sujet au monde (le «grand face-à-face », qui conditionne et intègre la confrontation intersubjective par la communication, le «petit face-à-face »), sert de base à l'organisation architecturée des systèmes grammaticaux : dans un système donné, comme la détermination du nom, dédiée à une fonction sémantique spécifique, toutes les formes (articles indéfini et défini) « saisissent » des prises de positions mentales interceptives précoces ou tardives, primaires ou secondaires sur «l'axe du temps opératif» qui sous-tend le parcours mental d'une phase à la suivante dans les étapes de la formation du sens, du plus simple au plus complexe. Cette vision processuelle et procédurale de l'inconscient «psychomécanique »du mentalisme langagier est étroitement liée à la notion janétienne d'automate psychologique même si cette relation n'a pas été pleinement explicitée à l'époque. Le TBR concerne dans des conditions analogues la production (par le sujet) des classes de mots en tant que profils de formatage abstrait des notions sémantiques initialement indifférenciées; ou encore les degrés d'élaboration de «l'image-temps» portée par les modes verbaux (modes impersonnels «initiaux» et stylisés », subjonctif intermédiaire, indicatif terminal). Ainsi, la psychomécanique modélise le contenu du sens en fonction de son degré d'élaboration dans la chaîne de production mentale de la psychomécanique, les formes verbales étant les reflets expressifs communicationnels, empiriquement rendus tangibles par l'actualisation sensorimotrice. La verbalisation, en principe, ne sert qu'à la transition du «plan de la représentation» (langue) à celui de «l'expression» (discours) et à la livraison communicative de marqueurs symbolisant les opérations sémantiques qui en ont déterminé la sélection : la forme incarnée (le "physisme ») reflète et affiche pour la perception interprétative d'autrui la spécificité du parcours «psychique » réalisé par le locuteur; le paysage apparent est emblématique des évènements géologiques qui l'ont engendré, et sa perception doit permettre la réinvention interprétative d'une dynamique analogue.

En principe, la relation sens / forme est la même que celle de la LC : les formes sémiologiques tangibles encodent ou transduisent des processus de conceptualisation, et ce qui oppose la PM et la LC concerne essentiellement le contenu de la conceptualisation et la modalité de sa mise en place, avec la médiation du TBR pour la PM et la dimension métalinguistique des actes de représentation, qui ne se veulent pas une copie stylisée du réel. Par exemple, pour la théorie des modes verbaux (chronogenèse), la séquence opératoire (i) mode quasi-nominal (infinitif, participes), (ii) subjonctif, (iii) indicatif, n'est pas motivée par des contenus de perception de l'espace ou du temps empiriquement vécus, elle schématise la progression de l'acte de conceptualisation du temps, pensé dans la durée que dure son propre développement.

En fait, la relation sens / forme chez Guillaume est plus ambiguë qu'on ne le dit souvent. D'une part, des analogies entre les formes sont considérées comme symptomatiques de la cohérence de l'architecture psychomécanique (par exemple la finale $-a$ de chantA et chanterA en dépit d'origines distinctes): l'existence d'éléments formateurs récurrents et cohérents préfigure une certaine vision de l'iconicité, même abstraite, d'une certaine coalescence forme / sens qui suggère que le profil de la forme n'est pas si étranger à celui du sens qu'on le dit généralement, quelle que soit l'orientation de cette relation (la forme comme reflet, ou comme vecteur, du sens). D'autre part, l'expérience du langage est ressentie comme essentielle dans la capture et la mise en ordre de la pensée «lucide »- comme si l'acte de signification était 
rendu possible par le vécu des actes de signification, même mentalisés dans le cas de la parole intérieure (endophasie) : même quand il ne s'agit pas de communiquer, la «pensée »doit, pour Guillaume, s'incarner dans des formes orales mentalisées pour « se saisir elle-même » et échapper à la «fugacité ». On constate (i) que l'ancrage de la conceptualisation au monde naturel est très indirect chez Guillaume, médié par le TBR, et (ii) que la corporéité du signe en tant qu'action mentale commence à poindre. Pour ces raisons, nombre de guillaumiens considèrent la LC «trop en prise avec l'extralinguistique » et incapable de modéliser la cohérence propre à la langue sans s'inspirer d'une vision naïve, matérialiste, positiviste, psychologisante, de l'expérience subjective du monde : la «langue » comme «système de systèmes de représentations » est porteuse de procédures psychomécaniques de régénération du sens à mettre en œuvre par «l'actualisation », homologue de la «performance » dans ce cadre théorique. Et si le profil de la mécanique intuitionnelle et du tenseur binaire radical sont indirectement motivés par l'expérience sémiotisée du monde par le sujet, il est clair que les systèmes grammaticaux et lexicaux n'encodent pas un modèle conceptuel emprunté au traitement psychologique de la perception non verbale par la catégorisation.

Pour ce qui est de la générativité, ou « actualisation du discours » à partir du plan puissanciel de représentation correspondant à la «langue », la psychomécanique est dépourvue d'une théorie de la syntaxe de la phrase en tant que schème de langue et que compétence générative : le sujet n'a aucune connaissance stabilisée du modèle phrastique à produire, aussi ce modèle n'a-t-il aucun statut théorique qui corresponde à un modèle ou une intention mentale. La raison en est simple : chez Chomsky, avec la pauvreté du stimulus et le caractère non répétitif des phrases produites, l'unité de base est la phrase minimale comme structure syntaxique abstraite, non instanciée par le lexique (vs les constructions de la LC) et non incarnée par l'action phonatoire; cette dimension intangible, insensible, ne pose aucun problème dans un modèle innéiste qui ne fonde pas l'acquisition du schème phrastique sur l'enregistrement mémoriel de l'expérience sensori-motrice, l'incarnation. Guillaume, au contraire, est naturaliste, et rien ne l'autorise à postuler un schème syntaxique mental, constitutif de la production des phrases, si rien dans l'expérience sensible n'en fournit le modèle acquérable et reproductible, expériençable et mémorisable. Le raisonnement guillaumien a été de considérer que la seule trace mémorielle laissée aux individus par l'expérience de la parole et des phrases, c'est le lexique catégorisé, l'ensemble des mots décontextualisés et organisés en classes, des fragments de phrases conservés comme pièces de puzzle formellement profilées en vue de réassemblages ultérieurs, originaux mais conformes à des procédures de recomposition. Il fallait donc élaborer une typologie lexicale prévoyant des caractéristiques formelles combinatoires contraignantes à partir desquelles les processus de recombinaison fassent mécaniquement émerger les structures phrastiques attestées à partir du seul savoir-faire procédural enregistré au niveau du mot et sans jamais évoquer un schème syntaxique mental correspondant au niveau $\mathrm{P}$ de la grammaire générative : en PM, tout le savoir-faire syntaxique génératif se réextrait des classes et de la morphologie lexicales, ce que résume l'aphorisme «toute langue a la syntaxe de sa morphologie ». Guillaume élabora l'élégante "théorie de l'incidence », qui caractérise les classes de mots en fonction des types de relations possibles et prévues au niveau du mot de langue en amont de son réengagement discursif; la typologie des régimes incidenciels est constitutive des classes de mots (nom, adjectif, verbe, adverbe), des classes de marqueurs grammaticaux par lesquels on retraite et paramètre certaines de leur possibilité à l'occasion du réengagement discursif (article, préposition, auxiliaire, conjonction) et des types de connexions syntaxiques possibles, au niveau du mot lexical de langue (adjectif $\rightarrow$ nom, adverbe $\rightarrow$ adjectif : incidence externe du premier / second degré) et du syntagme de discours (prédicat $\rightarrow$ sujet, quelle que soit la complexité des instanciations respectives). Dans le cas du français, ceci fonctionne bien : il est aisé de montrer que le potentiel combinatoire du nom et du verbe en tant que classes 
formelles des mots du lexique sont en effet les précurseurs des propriétés du sujet et du prédicat en tant que gabarits syntagmatiques constitutifs de la phrase sans en passer par la séquence NP + VP. Mais comme je l'ai montré dans diverses études, ce fait est précisément caractéristique du système indo-européen en général, et du français en particulier (en tant que langue à sujet obligatoire et conversion verbo-nominale morphologiquement marquée dans les deux sens), mais il n'est pas généralisable et ne s'applique pas typologiquement à des langues agglutinantes et à catégories lexicales souples ou inexistantes comme le basque, le tagalog (Philippines) ou l'inuktitut (Groenland occidental et îles arctiques), sauf à élaborer, comme le fait Guillaume, une «théorie des aires » particulièrement ardue, dans laquelle une modélisation très spéculative des rapports matière / forme dans le mot de langue joue un rôle moteur dans le type syntaxique des énoncés actualisables.

Il se trouve donc que la psychomécanique est un précurseur méconnu à la fois du générativisme et de la LC : du générativisme en tant que modèle d'un rapport compétence / performance, mais d'essence naturaliste fondé sur l'ancrage incarné de la formation de son schème principal, le TBR de la mécanique intuitionnelle; et précurseur de la LC (via la sémantique général de Pottier notamment) en tant que modèle de processus de conceptualisation, avec des formes grammaticales qui reflètent des architectures mentales, et un objectif scientifique qui se résume à des appariements de formes et de processus (de natures différentes pour la PM et la LC). Le générativisme, en réaction au behaviorisme et dans le contexte de la cybernétique et du cognitivisme émergents, a sans le savoir poussé à l'extrême un aspect de ce que devient la PM lorsqu'on la retranche du contexte naturaliste et incarné qui en fonde la racine, le TBR. Et la LC, en se rebellant contre le programme générativiste, a sans le vouloir réinventé un représentationnalisme de type guillaumien, dans un esprit naturaliste internaliste centré sur l'individu, mais l'attention prioritaire accordée au rapport direct moi / monde dans l'expérience individuelle et hors d'une formalisation générale de ce rapport a mené à la recherche de correspondances entre des schèmes conceptuels matérialistes et des structures phrastiques et lexicales (la constructique), écrasant au passage la distinction compétence / performance ou son précurseur guillaumien, le clivage langue / discours, représentation / expression avec, entre les deux, l'actualisation. Ainsi, la PM et la LC diffèrent par la localisation de «l'embodiment »: en LC, elle concerne la formation des catégories et images-schémas à encoder par le lexique et plus généralement la constructique, à savoir le supposé niveau prélinguistique que la langue encode; en PM, elle concerne la formation du schème cinématique général qui sous-tend tous les systèmes de langue envisagés comme chronologies opératoires automatiques de genèse et traitement du sens et non comme schématisations de l'expérience vécue par occurrences. Ce positionnement de la forme langagière au cœur même des processus sémantiques occasionne une ambivalence en PM que l'on ne trouve pas en LC: en LC, les formes constructiques observables reflètent l'organisation conceptuelle sans participer activement à leur mise en place. En PM, les formes (lexicales et morphologiques) «saisissent» les étapes des processus psychomécanique et, peut-être, y participent activement.

Il existe, en PM, une possibilité de corporéité cognitive au niveau même des signifiants de langue, en tant que formes coexistant au sein de paradigmes et que l'on peut rapprocher par l'analogie. Il s'agit d'une première étape vers la prise en compte du rôle de la corporéité du signe dans les processus de conceptualisation: un système grammatical peut évoluer historiquement vers plus de cohérence, de simplicité et d'efficacité représentationnelle lorsque les sujets qui en font usage remotivent analogiquement des ressemblances fortuites et forment à travers l'usage des paradigmes dont l'organisation semble résulter d'une visée intentionnelle, inconsciente et collective (la téléonomie). Mais il ne s'agit toujours pas de la seconde étape, celle qui consiste à considérer le rôle de l'embodiment dans la mise en œuvre 
même des formes de langue par la parole, la processualité de l'expérience articulatoire du lexique et de la morphologie et celle des enchaînements syntaxiques. Tournons-nous maintenant vers des modèles qui actualisent pleinement cette orientation en considérant la parole sous l'angle de l'expérience d'actions interactives situées dans une dynamique collective. Ces modèles s'inscrivent dans les paradigmes de l'enaction (Maturana 1978, Varela et al. 1993, Erard 1998, Kravchenko 2004, Auchlin et al. 2004, Bottineau 2010b), de la cognition distribuée (Cowley 2007 et 2009), de la biosemiosis (Hoffmeyer 2008), et ils se cristallisent dans la «grammaire instructionnelle» (Col et al. 2010) et la «théorie des actes corporimentaux langagiers » (Bottineau 2010c).

\section{Parole, corporéité et cognition distribuée}

\section{- Les trois personnes phénoménologiques de la relation langagière}

Pour sortir des apories que nous avons évoquées, il importe de reconstruire descriptivement le processus de la parole dans la diversité des points de vues phénoménologiques adoptables (Keller 2006), dont : celui du parleur ( $1^{\mathrm{e}}$ personne), celui de l'écouteur ( $2^{\mathrm{e}}$ personne), et celui de l'observateur ( $3^{\mathrm{e}}$ personne), typiquement le linguiste (ou autre) en position d'enquêteur expert. La distinction des trois points de vue est nécessaire pour spécifier le point de vue adopté à tout moment de l'exposé, mais elle est illusoire :

(i) le parleur est, à tout moment de la parole, son propre écouteur réflexif ; il improvise la poursuite de la parole en fonction de la semiosis des effets proprioperçus de sa propre production et de ceux de la réactivité de son écouteur en titre.

(ii) l'écouteur ne reconnaît et «comprend » ce qu'il perçoit qu'en mobilisant un savoir-faire pragmatique correspondant à l'activation de sa propre expérience de parleur. Depuis 1976 les expériences renouvelées sur l'effet McGurk montrent que la reconnaissance des syllabes passe par la simulation du geste moteur par lequel on produirait soi-même le signal " perçu », et que la captation de signaux contradictoires par voie visuelle et auditive peut induire la simulation d'une motricité hybride intermédiaire responsable de la «présentation consciente » d'une « image acoustique » ne correspondant ni au signal auditif, ni au geste visuel.

(iii) l'observateur ne peut lui-même s'empêcher de «percevoir » en simulant l'articulation des deux autres points de vue en mobilisant sa propre expérience de son engagement personnel dans les deux rôles. Les sommets du triangle phénoménologique ne valent que par les relations qu'ils entretiennent avec les deux autres par le jeu des codéterminations.

\section{- La corporéité entre exaptation et adaptation}

Du point de vue de l'embodiment, la parole est vécue par le parleur comme un engagement corporel improvisé et régulé, exactement comme une pratique sportive disciplinée telle que la gymnastique ou le tango. Cet engagement comporte des unités de base et des figures imposées: le lexique, sa variation morphologique, les morphèmes libres (mots grammaticaux), les enchaînements séquentiels de classes de mots ou groupes de mots (la syntaxe), et la prosodie (la variation accentuelle et tonale sur des segments dont l'extension varie de la syllabe à la tirade complète). Toutes les productions consistent à créer des effets syllabiques : une syllabe est la déviation de l'air expiré du canal nasal vers le canal oral, avec perturbation de la turbulence et génération d'un effet sonore intentionnel (connu, reconnaissable et rééditable). Cet effet est obtenu par coordination d'une matrice de gestes articulatoires réalisés par une coalition d'organes hétérogènes (lèvres, dents, palais, langue, pharynx, larynx, glotte, diaphragme selon les langues). Les organes recrutés par chaque matrice sont exaptés de leur fonction originelle (soufflement, succion, lèchement, morsure, 
cisaillement, mastication, déglutition, toux, crachement, vomissement ; de manière générale, relation, absorption ou éjection d'un corps étranger au système buccal) et adaptés autour d'une fonction secondaire fédératrice, (a) par recontextualisation (les organes interagissent entre eux au lieu de se concentrer sur un objet matériel d'origine externe) et (b) par coordination intentionnelle (la production des effets sensorimoteurs-sémiotiques recherchés) (MacNeilage 1998, Corballis 2003), avec des effets de contraintes réciproques. La production d'une séquence syllabique unitaire reconnaissable comme le mot «chien» induit la réactivation pour qui la perçoit d'un effet sémantique déterminé : un réseau de connaissances extraites des multiples occurrences discursives en contexte (cf. infra), ce qui confère à la notion culturelle l'hétérogénéité de sa diversité dialogique, au point d'incorporer des valeurs contradictoires $^{7}$. Toutefois, le lexique peut incorporer des éléments formateurs sous la forme d'agglutinations ou matrices consonantiques spécialisés dans la spécification d'une classe d'expérience sensori-motrice avec laquelle un sujet humain interagit typiquement avec l'entité ou l'évènement (Bottineau 2010a) ${ }^{8}$. Ces éléments formateurs stipulent une classe d'expérience sensorimotrice convenue par laquelle l'objet ou le processus évoqué est typiquement appréhendable, et ils fournissent un classement pragmatique efficace du lexique, permettant sa prolifération par variation sur thème sans compromettre l'intelligibilité de l'ensemble par dilution. Ces marqueurs sont bien culturels et non «naturellement déterminés » par une évidence empirique ${ }^{9}$. Par delà les études traditionnelles sur l'iconicité, la motivation et le phonosymbolisme, des modèles récents étudient l'ancrage biomécanique de la sémantique des «submorphèmes » et la dynamique diachronique de leur progression par transfert inter-domaine (Philps 2009).

\section{- La non-localisabilité des processus verbaux}

Dans l'expérience de la communication, où un parleur apparent semble adresser un signal à un écouteur apparent, il semble que le signal encode un sens intentionnel du côté du parleur et que l'écouteur saura reconstituer ou simuler par réappariement analogique de la forme et du sens dans son propre système de correspondances. En fait, une telle approche ignore l'endophasie, la parole intérieure réflexive, intentionnelle (la «pensée ») ou non (la « rêverie »). L'endophasie est très peu prise en compte en linguistique (Bergounioux 2004), son étude relève habituellement de la psychologie (Shanon 1993, Laplane 2000). Pour "penser », on ne peut faire autrement que rêver éveillé de sa propre parole, simuler ce que l'on dirait si on le «faisait » somatiquement, se faire vivre une aperception au moins partielle de ce que donnerait le couplage motricité / sensorialité. Evidemment, le simuler ou le réaliser, c'est la même chose. La pratique routinière de l'endophasie suggère que le sujet humain acculturé est entraîné à auto-focaliser son attention sur la production de contenus intentionnels qu'il se fait interpréter réflexivement en s'adonnant à la simulation de la boucle motrisensorielle de la phonation imaginée : ce faisant, il se fait «interpréter le sens » dans des conditions de réception interprétative analogues à celles qu'il soumet à un allocutaire distinct

\footnotetext{
${ }^{7}$ Exemple : «je ne vais pas être chien avec toi » vs « elle a du chien, cette nana »- les connotations divergentes, extraites de situations de discours contrastées, inscrivent dans la mémoire dialogique du mot des valeurs apparemment contradictoires hors contexte, et que l'on relie difficilement à la représentation de l'animal.

${ }^{8}$ Exemple: en anglais, les verbes monosyllabiques commençant par sw- impliquent tous l'expérience de l'oscillation, la pendulation, le balancement: swing «balancer», swim «nager », sweep «balayer», switch « commuter», et bien d'autres.

${ }^{9}$ Une notion comme l'écriture passe par des marqueurs différents dans les langues romanes et germaniques: écrire < scribere avec scr-associé à l'expérience de la gravure et de la friction ; vs write avec wr- associé à celle de la torsion (cf. wriggle «se tortiller», laurel wreath «couronne de laurier», wrath « rage », etc.). Le geste graphique dans sa complexité est susceptible d'être évoqué par des impressions partielles complémentaires (le gravage, l'arabesque) qui sont retenues de manière aléatoire comme autant de motifs concurrents et contingents dans les lexiques des langues porteuses de traces culturelles.
} 
dans le cas de la parole vocale somatisée et adressée. Ce seul fait oblige à considérer que la parole en tant que discipline vocale régulée est une mobilisation corporelle dont l'effet est de conduire l'avènement de productions sémiotiques à la conscience de «soi-même » et/ou « autrui », sans même qu'il y ait nécessairement discrétisation consciente et intentionnelle des deux entités, comme en témoignent les travaux de Vygotski sur le continuum psychologique intersubjectif des jeunes enfants ou, plus généralement, les multiples occasions où l'on parle sans que la question de savoir à qui on s'adresse ou si on s'adresse à quelqu'un, même « soimême ", ne soit pertinente : pour que la parole fonctionne, il n'est même pas nécessaire que cette opposition soit consciemment posée ; au contraire, la parole est l'un des médiateurs de soi à soi/autrui par lesquels se joue la co-constitution biosémiotique du sujet agissant et de l'objet «agi », lequel peut être réflexif.

La parole est incarnée parce qu'elle passe, par définition, par l'exécution d'une boucle motrisensorielle, somatisée ou simulée ; et elle est distribuée en ce qu'elle co-affecte soi-même et/ou autrui via l'environnement, les trois étant réalisés de manière compacte ou discrète selon le degré de décompression consciente de la triade environnementale par le ou les participants mobilisés. Ce paradoxe de l'incarnation intersubjectivement distribuée et coordonnée tant par le recrutement de l'environnement (propagation des signaux acoustique et optique) que par la synchronisation directe des corps en interaction (empathie, neurones miroirs) permet d'envisager la parole comme le vecteur corporel de processus sémiotiques pouvant concerner soi-même, autrui, les deux ensemble, indifféremment ou contrastivement. Dire ceci revient à dire, en termes binaires et localistes, que la parole est un moyen corporel d'agir sur l'esprit, ou, en termes d'unification, que la parole est la manifestation corporelle de l'autodétermination de l'esprit. Mais la terminologie est fallacieuse : d'une part, les processus biomécaniques porteur de semiosis sont sociaux tant par les modèles pris pour ressources que par les modalités intersubjectives de la mise en œuvre et la distribution intersubjective des effets épiphylogénétique, au point de destabiliser l'idée évidente selon laquelle la parole implique des partenaires discrets (comme dans les schémas de la communication de Bühler 1934 et Jakobson 1963 ou l'appareil formel de l'énonciation de Benveniste 1970).

Mieux vaut, au contraire, considérer la parlance comme un réseau processuel à la fois intégré et distribué, qui non seulement recrute et forme des participants-contributeurs dans l'instant et dans la durée, mais aussi renégocie continuellement les frontières diffuses ou discrètes de chaque élément, au point de requérir un principe de non-localisabilité du siège des processus décrits (Cowley 2010). Ce principe remet profondément en cause l'opposition intérieur / extérieur sur lequel se fonde l'opposition cognitivisme / behaviorisme (ainsi que, plus récemment, la cognition externaliste), mais aussi les approches qui corrèlent la dynamique verbale et syntaxique à la motricité neuronale (Allott 1995, Feldman \& Narayan 2004, Gallese \& Lakoff 2005). Dès lors que l'on réalise une construction phénoménologique fine de la distribution des processus incarnés et de leurs effets sur les corps et les consciences en instance de production conjointe, mais aussi sur les regroupements et dégroupements des individualités, on s'aperçoit que l'on ne peut plus raisonner en termes d'intériorisation des représentations : la LC, en dépit de son enracinement dans la psychologie, est fragilisée par une conception naïve de l'ego et du moi, de la stabilité discrète des « soi », de l'interiorité du mentalisme, de l'extériorité du physisme, de la médiativité du corporel, et de la séparabilité du fait langagier relativement au «reste » de l'expérience. Le «moi » est un effet plutôt qu'une cause, il s'obtient par des processus mentaux largement étrangers aux effets conscients induits (Frith 2007), et l'expérience de la parole joue un rôle moteur - littéralement - dans le processus, à la fois en imposant un protocole de conceptualisation appris de source externe par les interactions verbales, et en fournissant au sujet des modèles d'individualités centralisées auxquelles se conformer, comme l'appareil formel de l'énonciation de 
Benveniste. La parole suscite des «états du moi » thématisés qui, mis bout à bout et comparés à ceux d'autrui, finissent par synthétiser un «soi » crédible. Elle n'est pas la seule à le faire, bien sûr : toute confrontation du sujet au monde suscite des actes de perceptions consistant à produire des scènes mentales qui entrelacent le traitement des signaux captés par les récepteurs sensoriels ad hoc avec des attributions de propriétés hétérogènes tels que l'attribution émotionnelle de valeurs et la formation de projets d'action; et on sait que ces confrontations à caractère enactif, si individuelles et égocentrées paraissent-elles, mobilisent un système d'interprétation et de projection qui doit autant à ce que chacun a appris des occurrences antérieures dans le cadre des rapports sociaux qu'à un paramétrage matériel, objectif et naturel des choses du monde. Autrement dit, (i) il y a pensée sans parole et (ii) la pensée sans parole est imprégnée des savoirs socialement partagés avec et sans la parole. Dans ces conditions, on peut s'interroger sur la nature de la plus-value sémantique que représente pour le sujet un acte de parole effectif par rapport à un acte de «pensée » non médié par le protocole linguistique : faute de répondre à cette question, on retomberait dans le réductionnisme communicationnel et l'immotivation de l'endophasie. Pour y répondre, il faut repartir de l'expérience des unités fonctionnelles de la parole.

\section{- L'expérience « magdalénienne » du lexique : corporéité et réminiscence dialogique}

Du côté du lexique, dans l'expérience authentique et spontanée, un mot ne se présente pratiquement jamais comme un cri isolé et directement corrélé à un objet comme centre d'intérêt par l'action ou l'attention : a-t-on jamais vu une mère promener son bébé dans une pièce en pointant des objets et s'exclamant «Chaise! Table! Biberon! Papa!» pour dénommer chaque objet effleuré et mobiliser des couples signifiant / signifié ? Par contre, la mère parle à son bébé, sous forme de tirades, lesquels contiennent des mots, des segments non libres du discours. Tout mot dans son principe, en tant que catégorie ou ensemble d'exemplaires attestés et possibles, condense un ensemble de citations d'un extrait récurrent du discours de soi-même et d'autrui, citations dont l'unicité occurrentielle et épisodique est « oubliée » en fonctionnement normal, mais dont la trace sémantique, extraite du contexte phrastique et situationnel d'origine, est retenue, pour former cumulativement un réseau notionnel hétérogène de profondeur intertextuelle et dialogique ouverte. Son articulation par la boucle motri-sensorielle a pour effet de convoquer ce réseau dialogique par son historique interactionnel et normé par la prescription des classes d'interactions auxquelles il est réservé, implicitement (par l'usage) et/ou explicitement (par l'éducation et l'instruction). L'homogénéité intersubjective des réalisations individuelles de cette sommation dépend de la croyance que l'on a en la relative convergence des interactions verbales auxquelles chacun est exposé (on conçoit ici l'importance normative de la scolarisation institutionnalisée et des pratiques rituelles, notamment religieuses ; de manière générale, de la diffusion démocratique d'un discours expert fédérateur). Ceci fait du mot une «madeleine » sociale et normative : un geste buccal non pas accidentel et à effet aléatoire, mais volontaire et à effet intentionnel ; une corrélation geste / souvenir non pas fortuite, mais fournie en modèle contraignant (cf. les jeux littéraires de Lewis Carroll sur le sécessionisme métalinguistique) ; non pas idiosyncratique, mais fédératrice; un geste commun capable de susciter la réminiscence d'un réseau d'associations d'idées formé dans l'historique des interactions, avec oubli de l'épisodicité des interactions particulières et construction évolutive d'une synthèse qui synchronise et hiérarchise le tout, que Culioli 1981 nomme «notion », «ensemble hétérogène de propriété physico-culturelles » dont l'hétérogénéité reflète celle des interactions par lesquelles les «propriétés » se recrutent; et dont Rastier formalise la componentialité et la mise en réseau discursif dans le cadre de la sémantique interprétative. La sémantique lexicale résulte de la synthèse de l'ensemble des interactions en lesquelles une unité donnée est mobilisée, cette 
synthèse étant relative au point de vue de qui la réalise - d'où la nécessité de normes expertes, prescriptions académiques et inventaires lexicographiques.

En principe, la sémantique lexicale, de contenu «notionnel », diffère de la sémantique catégorielle de l'expérience non verbale, qui fédère des concepts sur la base de synthèses d'occurrences d'exxpériences non langagières. En pratique, cette distinction artificielle ne tient pas, justement parce que la parole, expérience sensorimotrice parmi toutes les autres, est de même nature que les « expériences » constitutives des catégories. Il n'empêche que le mot lexical pointe des trains de citations multiples et hétérogènes, et fournit une base gestuelle à la convocation de synthèses de connaissances culturelles qui, sans ce geste, seraient irréalisables. A ce titre, il fonctionne comme «amplificateur d'intelligence » au sens étymologique. La parole déforme intensionnellement le monde énacté par le sujet dans le cadre des pratiques de groupe, et ne voir en elle qu'un rôle de témoin de la conceptualisation relève du contresens.

\section{- Biosémiosis lexicale normative et transgressive}

La LC, en ignorant la corporéité du signifiant et l'intersubjectivité de cette corporéité socialement fédératrice dans l'interaction, se concentre sur la capacité du signe à encoder des catégories appréhendées à travers des actes de perçaction individuelle et autonome, non liée à la parole, ou très indirectement (par le jeu de la prescription verbale des affordances : «ne touche pas, ça brûle »). Or, on ne compte pas les enfants accusés de «manger comme un cochon » bien avant que le «cochon » leur ait été soumis dans l'expérience par un exemplaire bien en chair ou sa représentation en image (livre, dessin animé). Le mot «cochon » est pour eux un fragment récurrent de discours maternel réactif en situation spécifique : la récompense verbale pour une action alimentaire mal conduite. Certains mots ne valent presque que par la classe d'interaction dont ils sont extraits : «Alors, tu la gares, ta bouse ?» - inutile de rechercher la métaphore, on sait en situation qu'on parle d'une voiture et on n'a nul besoin de la désigner par un terme conventionnel, aussi peut-on remplacer le mot par la désignation d'une entité qui inverse la polarité des valeurs - «tu gares ce qui pour toi est, j'imagine, une «voiture », avec la valeur sociale que tu lui attribues, mais que moi je redéfinis comme une «bouse », objet laid, informe, malodorant, répugnant et sans valeur, avec la valeur de substitution que tu imagines, pour ton plus grand plaisir». Ce type de transgression est luimême régulé par l'usage, et on ne pourrait remplacer «bouse » par «étron » ou «crottin » et être compris. On ne descend pas pour «s'acheter un canard », mais on peut s'énerver contre « ce canard qui raconte toujours les mêmes catastrophes ».

\section{- La morphosyntaxe et la constructique, direction vocale des parcours biosémiotiques}

Du côté de la «grammaire », la parole mobilise deux types d'éléments : une morphologie, ensemble de marqueurs abstraits dont l'effet et l'activation de classes profilées d'actions de repérages spatiaux, temporels, psychologiques, intersubjectifs, discursifs, et de classes de connexion sémantique entre entités lexicales; et une syntaxe, ensemble d'enchaînements comportementaux de mots ou groupes de mots correspondant à des étapes routinières de la convocation du sens, typologiquement caractéristiques d'une langue donnée, et formalisables comme le fait la grammaire instructionnelle (en repartant des notions culioliennes de forme schématique et de scène verbale). L'idée que la syntaxe doive être modélisée comme un processus est encore peu répandue : Auer 2009 la défend pour la langue parlée, par opposition à la langue écrite, oubliant le fait que la lecture du texte écrit nécessite une reconstruction processuelle en syntaxe. Ici encore, la marche à suivre dans le «faire sens » s'apprend dans le cadre de la pratique interactive des modèles communautaires, dont les contenus sémiotiques peuvent être précisés par la sémantique interprétative. Par exemple, en français, la séquence SN - SV, obligatoire même en cas de vacuité sémantique du sujet (cf. les énoncés 
impersonnels : il pleut), correspond à une procédure d'amorçage attentionnel de la réception de l'effet en passant par un relai initial censé réactiver une entité connue et intersubjectivement partagée dans le cadre de l'attention conjointe : on part de ce autour de quoi les attentions sont déjà conjointes et on progresse vers ce sur quoi elles doivent encore le faire, du point de vue du parleur du moins.

En breton, c'est l'inverse : l'amorce préverbale reçoit l'entité lexicale ressentie par le parleur comme figurant le segment de sens sur lequel les attentions doivent se focaliser immédiatement (Didier eo ma anv 'Didier est mon nom' $\rightarrow$ «je m'appelle Didier »). L'amorce peut recevoir le sujet, l'objet, l'attribut, un circonstant, au gré des sélections intuitives du locuteur, et la structure de conjugaison verbale s'ajuste de manière plastique en fonction de l'amorce qui le précède. A cette focalisation syntaxique correspondent dans d'autres langues (wolof, tagalog) des focalisateurs morphologiques infixés au verbe : un effet d'orientation attentionnelle comparable peut être obtenu par une procédure morphosyntaxique très différente, et la mise en contraste des éléments de description typologique permet de rendre compte des spécificités et homologies tant dans la démarche procédurale que dans le profil des effets sémantiques, psychologiques, intersubjectifs, qu'elles permettent de produire.

En basque, la procédure consiste à convoquer les arguments nominaux avant de les engager dans le «procès » par la conjugaison agglutinante du verbe ou auxiliaire final : Haurrek pastela jan dute 'enfants-les (ERGATIF : agent) gateau-le (ABSOLUTIF : patient) mangé le-ils' $\rightarrow$ «Les enfants ont mangé le gateau ». Les participants énumérés reçoivent des marques « casuelles » (ergatif, absolutif) qui leur assignent des rôles dans la scène en construction (ici, respectivement : saillance, base), puis sont confrontés en relation à l'évènement qui motive leur mise en présence dans une tirade commune. Ainsi l'enfant en milieu bascophone est-il confronté à l'expérience répétée de cet algorithme qui s'impose comme un cycle biomécanique commun de conceptualisation, une horloge mentale récurrente profilée par les routines verbales, et il apprend à le pratiquer, pour autrui (exophasie) comme pour lui-même (endophasie), en conjoignant sa reproduction phonatoire et interprétative.

On comprend que chaque type linguistique se caractérise par son horloge syntaxique particulière : une séquence opératoire spécifique qui se répète de manière variable, comme une variation sur thème. En parlant une langue donnée au contact d'autrui, on apprend à générer des synthèses sémantiques, pour soi-même comme pour autrui, en recourant aux notions dialogiques stockées par son lexique (en tant que fragments de discours des membres du groupe parlant cette langue) et aux protocoles morphosyntaxiques de combinaisons et de repérages, et on forme son esprit à « penser » en respectant ce protocole plutôt qu'un autre. Le bilinguisme suppose la pratique de cycles contrastés, comme ceux du basque et du français ; l'apprentissage d'une langue étrangère, la découverte d'un cycle distinct de celui de la langue première ; la traduction, la transition d'un cycle à un autre, via des procédures ad hoc qui ont été décrites par la stylistique comparée comme celle de Vinay \& Darbelnet 1958. En abordant la syntaxe comme un jeu muni de règles de procédure et d'une finalité, on peut faciliter la découverte d'une langue exotique en explicitant la nature de la démarche qu'il s'agit de s'approprier (exactement comme on enseigne les échecs en commençant par les fins de parties avec le moins de pièces possible): en breton, pour «parler », on focalise l'attention de l'interprétant sur un élément ciblé auquel on raccorde des ajouts via le verbe ; en basque, on présente un à un les éléments comme un casting cinématographique ou dramatis personae théâtral avant d'engager l'intrigue par le groupe verbal final ; en français, on part d'une plateforme reconnue, le sujet, pour y introduire des modifications. Pour expliquer l'article français à un Russe ou un Japonais (Bottineau 2010d), on lui apprend à avertir l'allocutaire (= opérer un amorçage, priming) qu'on va lui présenter une notion lexicale correspondant à une occurrence reconnaissable (= définie: le livre) ou originale (= indéfinie: un livre), 
exactement comme on sonne à l'entrée d'un bureau en utilisant le timbre grave si on est de la maison et le timbre aigu si on est un visiteur; et on explique à l'apprenant qu'à défaut du respect de ce protocole, l'interprétant est brusqué parce qu'il est entraîné à ce qu'on lui présente les notions en suivant cette routine et pas autrement, que cela semble nécessaire ou non pour la bonne compréhension de l'ensemble : l'article n'est pas une nécessité cognitive ou représentationnelle nécessaire pour le sujet mais un habitus pour le protocole biosémiotique de la relation intersubjective - une procédure efficace, non nécessaire, mais inévitable en tant que rituel. Le japonais n'a pas d'article, mais un système de registres verbaux (informel, distant, honorifique) qui sélectionnent des profils de hiérarchisation des interlocuteurs en les marquant de manière distribuée sur la forme du verbe final et une partie du lexique nominal qu'il sélectionne en amont, avec ici aussi un effet d'amorçage diplomatique.

Dans tous les cas, apprendre ces langues, c'est apprendre ce que l'on fait conjointement à l'autre et à soi-même en produisant les constructions protocolaires dans leur dimension articulée et scénarisée, avec leurs effets sémiotiques; ceci oblige à s'écarter de la perspective encodagiste : la tirade n'éveille pas un schème représentationnel, elle produit un effet dont il faut modéliser le devenir et le contenu. Ceci apparaît sans doute pour la première fois dans Valin 1981 en syntaxe psychomécanique et devient systématique en linguistique instructionnelle avec la scène verbale, et avec la théorie des actes corpori-mentaux langagiers. Cette approche permet de rendre compte de la compétence procédurale des sujets, en tant que boucle biosémiotique de production et d'interprétation, avec ses effets spécifiques, sémantiques et psychologiques. Elle explique également nombre de caractéristiques systémiques des langues, comme, dans le cas du basque, la prohibition des constructions réfléchies dans les conjugaisons à double accord du verbe (Bottineau \& Roulland 2007) : on ne peut présenter successivement le même argument nominal deux fois en lui assignant deux rôles distincts, ni engager dans le verbe les indices correspondants. Le blocage ne résulte pas d'un problème représentationnel (le réfléchi existe dans bien des langues), mais d'une impossibilité procédurale modélisable. On a modélisé dans des termes analogues l'ergativité, la plasticité de la conjugaison allocutive et des structures impersonnelles en basque, les subordinations verbales en série du breton, et bien d'autres faits dans des langues diverses, impossibles à énumérer ici.

Plus généralement, dans le cadre de l'approche biosémiotique et instructionnelle, l'objectif de la description d'une «langue » devient l'inventaire de l'ensemble des procédures verbales de génération des effets de sens qu'elle mobilise, en combinant les scenarii syntaxiques, les activations lexicales (dialogiques), les activations morphologiques (relationnelles), les pilotages prosodiques de la coordination intersubjective. Une «langue orale » est un ensemble épilinguistique de pratiques spontanées en dynamique d'autorégulation démocratique sans contrôle des profanes par des experts autoproclamés en situation de pouvoir; une "langue écrite » est un ensemble de pratiques spontanées corrigées par un appareil métalinguistique de prescriptions expertes rendues possibles et nécessaires par les besoins de la fixation et conventionnalisation de la trace graphique, avec conflit permanent entre la force de dispersion que suppose la spontanéité orale et la force de coordination requise par l'écrit (et la destabilisation de ce rapport par les nouvelles technologies). Un «dialecte », comme le gallo parlé en milieu rural, est un ensemble relatif de pratiques spontanées reconnues propres à une communauté (locale, munie d'un mode de vie) aux yeux des autres communautés; une « langue » est un ensemble universel de pratiques verbales régulées et libérées de la signature sociolinguistique et culturelle d'un groupe identifiable (hormis celui des experts qui s'arrogent la normativisation). Le breton co-existe sous ses formes dialectales rurales culturellement colorées et sous diverses normes expertes comprenant un lexique universel (il 
est possible de construire une centrale nucléaire en parlant exclusivement en breton et sans emprunter les termes romans). Ce breton général est «culturellement incolore » relativement aux dialectes, mais porteur de la coloration culturelle mythique hétérogène que l'on peut lui attribuer en France selon les points de vue, de l'intérieur (par les brittophones : breton langue maternelle) comme de l'extérieur (par les francophones) dont les médiateurs (les bretonnants : breton langue seconde). La description des pratiques lexicales, morphosyntaxiques, prosodiques, discursives, dialogales se fonde autant sur la relativité culturelle des milieux locaux que sur l'universalité émergente de leurs rapports avec l'environnement général, dont la République Française centralisatrice et la pensée unique mondialisée via l'anglais.

La linguistique contrastive permet de comparer ces ensembles de procédures en détaillant ce en quoi deux langues se ressemblent ou diffèrent, et la typologie soulève la question de savoir si on peut repérer des universaux de processus biosémiotiques derrière la variation constatée une grammaire universelle incarnée si on veut, déjà envisagée par Guillaume sous la dénomination «grammaire générale » à caractère «panchronique ». Chacun des ensembles procéduraux constitutifs des «langues» a sa cohérence systémique propre, figeable par description et prescription experte et autoritaire, mais ouverte à l'hybridation du fait de la pratique de la diglossie; aucune de ces cohérences n'est nécessaire ni motivée (au sens de déterminée), et les sujets n'ont pas à les inventer, tout au plus à les sémiotiser correctement à travers leur expérience de la mise en œuvre incarnée et à s'entraîner à interagir correctement avec autrui dans une langue donnée en pratiquant régulièrement les normes protocolaires et diplomatiques dont les procédures sont porteuses, avec les effets ethnopsychiatriques que cela suppose (Denez 1986). En breton, il est impossible de s'adresser à autrui sans sélectionner comme amorce l'élément lexical par lequel on considère inconsciemment que l'on va obtenir l'effet recherché (émotionnel, pragmatique, théorique), alors qu'en français cette sélection est optionnelle et passe à l'oral par les détachements en amont du sujet (Le gateau, moi, je n'en veux pas) et produit un effet différent, une simple présentation introductive. En japonais, on ne peut formuler un verbe sans préciser le statut hiérarchique de la relation intersubjective que l'on affiche pour soi comme pour autrui par le jeu complexe des marqueurs de registre, lesquels sélectionnent à leur tour le lexique nominal et verbal co-occurrent, avec les subtilités et la délicatesse contraignante que l'on imagine. Ladmiral et Lipiansky 1973 ont nommé « incorporation » l'apprentissage épiphylogénétique de modes d'existence psycho-interactifs discrets par la pratique de la polyglossie, et l'écrivain Alexakis a produit plusieurs romans (dont Les mots étrangers) mettant en scène des personnages qui se construisent et se font évoluer par l'expérience multilinguistique et interculturelle. Le modus operandi de ces modes d'auto-détermination psychologique concerne le lexique et la phraséologie (les clichés, idées reçues, idéologies, prêt-à-penser) mais aussi et surtout son enracinement au cœur de la «grammaire» sous la forme des processus intersubjectifs de base à convoquer obligatoirement dans toute tirade.

On comprend donc que la parole se laisse interpréter comme un processus de coordination mentale selon un code social - code comme ensemble de pratiques régulées, comme dans « code de la route ». La grammaire au sens le plus large (lexique, morphosyntaxe, prosodie) est le code de la voix. La parole permet l'accès à des états mentaux intersubjectivement coordonnés et de contenu proprement verbal: les classes conceptuelles dialogiques historiquement construites par les pratiques verbales communautaires dans le cadre général de l'histoire socio-culturelle, les protocoles d'agencement et d'interconnexion en situation (y compris la rhétorique et la logique comme mode social d'argumentation et de raisonnement). La coordination concerne la coalisation ou l'amalgame intersubjectif des consciences «à l'externe », mais il concerne aussi la «coordination interne» de l'ego du sujet, qui se constitue en temps réel par ses projets d'action et leurs effets codéterminatifs sur la relation 
sujet / objet : la parole est l'un des instruments de la fabrication constamment renouvelée du moi, avec les effets inhérents à la parole - la conceptualisation de soi (émotions, actions) dans les termes collectifs permis par le lexique et la grammaire. La nature du sens ne va pas de soi, la nature des causes et effets de la parole ne vont pas de soi, le rôle de la parole, des langues et du langage dans l'homonisation immédiate et prolongée restent à ce jour très mal connus, et il est urgent de considérer la parole comme un symptôme de l'exercice du processus dynamique distribué qu'est «l'humanité », constitutif simultanément des sujets, des groupes, de l'espèce, des relations, de la civilisation, de l'histoire, des cultures, des psychologies. Ce programme est réalisable en partant d'une approche biosémiotique du rapport individuation / sociétalisation vectorisé par l'expérience de l'incarnation coordinatrice et fédératrice. Il suppose la mobilisation des paradigmes unificateurs du rapport corps / esprit, individu / groupe / environnement, et la relativisation du rapport intérieur / extérieur par la relation. Actuellement, deux paradigmes au moins sont en bonne position pour fournir les outils conceptuels, terminologiques et protocoloaires adaptés : la biosemiosis dans le cadre de la cognition étendue et distribuée, en particulier avec le principe de non localisabilité ; et le paradigme de l'autopoïèse et de l'enaction ${ }^{10}$ développé par Varela et Maturana; et divers travaux, que l'on ne peut évoquer ici, ont commencé à fournir des résultats précis en typologie linguistique, en sémantique lexicale, en morphosyntaxe, en prosodie, en phonologie articulatoire, mais aussi en sociolinguistique et en pragmatique, avec notamment l'étude de l'impact des nouvelles technologies (texto, réseaux sociaux...) sur les dynamiques psychosociales incarnées.

\section{Conclusion}

Le biologiste Chilien Maturana explique comment une espèce se couple à un environnement en développant évolutivement et co-adaptativement un «domaine consensuel d'interactions », lequel peut être ramené à une version fortement améliorée de l'Umwelt de Von Uexküll 1922 et 1934 dans le cadre de la phénoménologie merleaupontyenne et la théorie piagétienne de la connaissance: une sélection de signaux physiques réalisée par des capteurs biologiques, traitée par un système nerveux en fonction de son organisation et ses critères de tri (invention des couleurs, des formes), mais aussi de l'expérience enregistrée (incrustation du champ gravitationnel invisible dans l'image visualisée; attribution de valeurs pragmatiques, théoriques, émotionnelles, économiques à toutes les composantes). La mise en œuvre coordonnée de cet ensemble complexe de dynamiques de natures hétérogènes permet à l'être vivant de produire pour lui-même le sentiment de l'expérience d'un «monde » intelligible, où il est possible de se faire accéder à "l'actance » (l'état d'être agissant, "l'agissance si on veut) en tant que corps parmi d'autres reconnus tels, évoluant dans un espace construit et appréhendé comme espace. Ainsi, cette «caresse à distance » que constitue le geste oculaire, pour reprendre l'expression de Merleau-Ponty, entraine-t-elle parfois dans la conscience une véritable confusion entre l'objet du monde visuel et la sélection de ses propriétés par le regard, comme le montrent les expériences récentes d'Olivier et al. (2001). L'essentiel de cette mise en scène est illusoire à tous les niveaux (sensations, mémoire : Rosenfield 1997 ;

\footnotetext{
${ }^{10}$ «La théorie de l'enaction formalise l'idée d'une co-construction historique du sujet et de l'objet de la connaissance émergeant de l'interaction sensori-motrice d'un système vivant et de son environnement. Elle autorise, en outre, une conception de la connaissance de la réalité qui permet de repenser en termes conceptuels la question de la relation mental / physique, et de proposer une pratique d'articulation des comptes-rendus phénoménal et objectif, produits respectivement en première et troisième personne : la neurophénoménologie » (Peschard 2004).
} 
Frith 2010 : «notre perception du monde est un fantasme qui coïncide avec la réalité »), mais pragmatiquement efficace pour tous, et communément acceptée comme vraie ou réelle. Berthoz 2009 nomme simplexification ce processus dynamique complexe par lequel l'être vivant produit une présentation simplifiée et viable du monde physique, ce «X-monde» inaccessible et inconnaissable en amont de sa simplexification, et dont le «monde simplexe » s'inspire sans en être la représentation : non, la terre n'est pas plate ; non, le soleil n'est pas un disque aux bords tranchants, il ne se lève pas, ne se couche pas, n'a pas la couleur qu'il prétend, ne se trouve pas là où il se montre, et n'est pas plus petit que la lune : cette ontologie «substitutive » se présente ainsi parce qu'elle fournit une solution cohérente, efficace et satisfaisante à l'ensemble des symptômes collectables par notre système sensoriel (incapable, par exemple, de corréler le décalage vers le rouge de la lumière du couchant à la courbure du rayonnement solaire occasionnée par la pénétration atmosphérique suivant un angle presque tangeant : qui «voit » que la lumière a été courbée avant de nous atteindre et que le soleil est ailleurs ? et du point de vue de la survie, à quoi cela nous servirait-il de le voir, puisque jamais nous n'aurons à interagir avec le soleil là où il se trouve (sauf à le recréer localement par une explosion nucléaire) ? C'est le paradoxe bien connu du «pilotage aux instruments »: la perception ne représente pas le X-monde par un monde stylisé, elle crée un monde, un « gentil masque » posé sur le «méchant visage invisible » du «véritable »X-monde qui, sans cela, ne se manifesterait à nous que par l'effet-neige caractéristique des écrans de télévision non raccordés à une antenne qui sélectionne les bons signaux - tout voir, c'est ne rien voir, et mourir, ou ne jamais accéder à la condition d'être viable et effectivement vivant: le représentationnalisme internaliste est une impossibilité fatale, un scenario qui, par sa réalisation, ferait barrage aux exigences de la « vivance ».

Or la parole, par définition, est une intervention physique dans le monde, dont les propriétés percevables et biosémiotisables en boucle se trouvent modifiées dans l'instant, selon un protocole opératoire collectif, social, fourni par des modèles experts, « conventionnel ». Par la motricité, le parleur introduit dans l'X-monde physique environnant (invisible, non perçu, et relativement conçu par la physique) des perturbations que l'écouteur (réflexif et/ou adressé) « perçoit », réintroduit dans le «monde » qu'il produit (enacts) par simplexification au second degré. L'acte de langage vocal ne laisse aucune trace stabilisée et se limite au signal acoustique avec l'ensemble limité de procédures qui le profile et ses effets individuels, intersubjectifs et collectifs; l'acte de graphique stabilise une trace matérielle dans le «monde » perçu, profilée par le même ensemble de pratiques (lexique, morphosyntaxe) sous une réalisation adaptée aux conditions d'expérienciation de l'acte graphique avec ses spécificités (que Goody 1979 nomme «raison graphique »). Par son exécution, la parole vocale et scripturale représente l'inscription motrice de l'agentivité humaine dans l'environnement médiateur, avec la diffusion perceptuelle distribuée que cela suppose : la parole, en tant qu'action incarnée, se fond dans le monde qu'elle réforme. Par sa perception, la parole vocale et scripturale se distingue, comme processus et comme objet, de l'environnement et des supports : elle s'impose comme une Gestalt et focalise l'attention sur la production d'un «sens » dont on a montré la profondeur dialogique et non représentationnelle, ou, plus exactement, indirectement représentationnelle selon une synthèse de points de vue multiples. Le mot «table», par sa profondeur dialogique, synthétise l'ensemble des savoirs que chacun a pu extraire de l'ensemble des discours où s'en manifeste un exemplaire contextualisé par une prise en charge incarnée dans une situation interactionnelle et environnementale. De ce fait, le mot «table» représenterait une opération de simplexification du second degré par rapport au premier degré que représente la perception visuelle (avec son appel à la mémoire pragmatique des rapports antérieurs à aux tables); le langage corrige le «percevable» en $\mathrm{y}$ incrustant biomécaniquement des accidents sémiotiquement pertinents et, ce faisant, il refonde le réel. 
Il faut donc comprendre qu'à l'interieur du domaine consensuel d'interactions propre à l'humain (Maturana 1978), il se manifeste un sous-domaine conventionnel d'interactions spécifique, la «parole», manifestation d'une compétence biosémiotique. Ce sous-domaine particulier s'incruste dans le domaine général par l'action motri-sensorielle et constitue un sous-système simplexe de «perçaction» capable d'affiner le système général de la perçaction. Son effet est de permettre une simplexification complémentaire, une semiosis de second ordre qui s'articule par rapport à la première selon un rapport ambigu : distinctif et séparatiste par la singularité gestaltique et la focalité attentionnelle de ses manifestations incarnées et matérialisées dans «l'extériorité », mais fusionnel par sa capacité à rectifier notre perception / dramatisation du monde plutôt qu'en produire une représentation parallèle diplomatique qui serait assumée comme telle et ressentie comme virtuelle, fictive, voire mensongère. L'effet de la parole est d'amener les consciences coordonnées à une conscience focalisée et fédératrice de l'expérience vécue, diversement reliée à la situation matérielle (dont la parole elle-même), et obtenue selon un protocole commun, la «langue » comme système de production biosémiotique autorégulé, formalisme procédural collectif individuellement incarné.

A l'analyse, il est nécessaire de distinguer les semiosis de premier ordre (perception / action / émotion, mémoire, catégorisation sémiotique), relatives aux rapports individus / objets dans le monde, des semiosis verbales de second ordre, relatives aux rapports intersubjectifs codifiés par rapport à des contextes situationnels, pragmatiques, intellectuels, émotionnels impliquant indirectement des objets: la semiosis de premier ordre semble individuelle, la seconde collective et régulée par un code social. Mais ce distingo n'est utile que pour spécifier ce que le langage apporte par sa mise en œuvre. Dans la « réalité », à aucun moment on n'interagit avec des objets matériels sans que la mediation verbale n'en affecte la semiosis, immédiatement en cas d'interaction concomitante, ou par le biais de la mémoire, qui réactive continuellement, sous la forme des affordances, les connaissances pragmatiques relatives aux objets, acquises par voie interactionnelle et pragmatique indifférenciée dans le vécu antérieur. Je sais que je sais que l'amanite est mortelle parce qu'on me l'a dit et non parce que j'y ai goûté, mais qui des profanes est conscient de la source des savoirs mobilisés ? Donc: la parole, sous domaine d'interaction conventionnelle, évidemment incarné dans sa praxéologie signifiante, porteuse d'un second degré socialement codifié de simplexification, capable d'intervenir sur le premier degré plus spontané (aussi social, mais non codifié) sans véritablement s'en distinguer; la parole, moteur naturel culturalisant de l'engagement au monde, dans sa double relation de réforme du monde et de réforme des consciences, avec effet épiphylogénétique de la formation des consciences individuelles, interactionnelles et collective, une géométrie et discrétisation variable des participants recrutés par le réseau, une covariation de la nature et portée des effets, et un principe de non-localisabilité des processus. Dans ce réseau, la corporéité (embodiment) est l'interface motrice et sensorielle par laquelle le sujet participe bilatéralement au processus général, d'une part en inscrivant ses contributions particulières aux effets généraux émergents, d'autre part en incorporant à sa propre structure et pour son propre bénéfice l'expérience extraite de sa présence au réseau, tant dans sa dimension générale conformatrice (l'hominisation) que dans sa dimension particularisante (l'autopoïèse individuatrice). Par cette relation bilatérale, la corporéité cospécifie ses termes, l'individu et le groupe, garantit l'intégration réciproque et évolutive des propriétés dynamiques de chacune dans l'autre, et vectorise leurs devenirs solidaires aux différentes échelles spatio-temporelles.

Cette définition volontairement omnidisciplinaire implique le recrutement et la coalisation de participants disciplinaires spécialisés tels que la biologie, la philosophie, la psychologie, l'anthropologie, l'éthologie, la sociologie, les sciences du langage, les sciences cognitives, 
etc. Elle fait de l'embodiment le constat heuristique plutôt que la théorisation, et elle vise à rendre explicitables toutes les conséquences de cet ancrage phénoménologique dans la médiation.

C'est précisément le travail que n'a pas réalisé la LC, qu'a amorcé la PM, et qu'a exclu par principe le générativisme; et c'est cette insuffisance qui conduit directement au représentationnalisme innéiste incarné par les ICM Lakoffiens (Modèles Cognitifs Idéalisés), au «psychologiquement réel », ou au computationnalisme modulaire désincarné. En raison de sa méthode, de l'objet qu'elle s'est donné, des objectifs qu'elle se fixe, la LC s'assimile davantage à une psychologie assistée par le langage et les langues qu'à une linguistique cognitive à proprement parler. Toute la question est de savoir s'il est légitime de prendre les langues, fait collectif, comme symptômes de processus psychologiques internalisés et individuels, et si, inversement, l'organisation des langues s'explique par ce motif. L'externalisation de procédures délocalisées reproductibles permet, en principe, de dispenser le «cerveau » et «l'esprit » d'enregistrer des connaissances inutiles toujours réacquérables (Havelange, Launay \& Stewart 2003), même si le renforcement des interprétations (entrenchment) permet à la mémoire de stabiliser certaines représentations. La question est alors celle de la redondance, qui n'est plus considérée comme un défaut des systèmes cognitifs. L'amalgame élégant «Grammar is conceptualization », jouant sur la polysémie très ouverte des deux termes mis en présence, masque une constellation de problèmes plus qu'il ne les résout.

Tout l'enjeu est de définir l'ensemble des processus constitutifs de ce rapport ambigu, leur variation interlinguistique, leur participation à la coévolution du monde-homme et de l'homme-monde. Le projet semble plus réalistement ambitieux que celui de décrypter les représentations encodées ou formalisées par la constructique, il donne les moyens d'accéder aux enjeux les plus fondamentaux pour la survie et le bien-être, et il en passe par la fondation d'une biosemiosis incarnée et distribuée, enactive et étendue, autopoïétique et non localisable. Au bout du compte, en replaçant la corporéité des actes de signification à la racine empirique $\mathrm{du}$ fait langagier, on contraint fortement le contenu des hypothèses qu'il est légitime de formuler. Notre position est que les gestes de la parole ne représentent pas des processus cognitifs, ils sont le symptôme du rôle de la boucle corporelle dans leur exécution, et cette approche repose la question du sens, qui n'est pas une donnée de départ, mais un aboutissement.

\section{Références}

AllotT R. 1995. "Syntax and the motor theory of language". In Syntactic iconicity and linguistic freezes, ed. M. E. Landsberg, 307-329. Berlin: Mouton de Gruyter.

Auchlin A., Filliettaz L., Grobet A. \& Simon A. C. 2004. «(En)action, expérienciation du discours et prosodie ». Cahiers de linguistique française 26, 217-249.

AUER P. 2009. On-line syntax: Thoughts on the temporality of spoken language. Language Sciences 31, 1, 1-13.

BAKHTINE M. [1929] 1977. Marxisme et philosophie du langage. Paris : Minuit.

BANG J. C. \& DøøR J. 2007. Language, Ecology and Society - a dialectical approach. Ed. by S. V. Steffensen \& J. Nash. London: Continuum.

BARBIERI M. 2007. Introduction to Biosemiotics:The New Biological Synthesis. Dortrecht: Springer.

BARSAlOU, W. L. 2008. «Grounded cognition », Annual Review of Psychology, 59 : 617-45.

BEGIN, L. 1998. Reconstruire le sens de sa vie, Le changement thérapeutique, Montréal : Éditions Nouvelles. 
BENVENISTE E. 1970. «L'appareil formel de l'énonciation ». Langages, 17, L'énonciation, 1218.

Bergounioux, G. 2004, Le moyen de parler, Paris : Verdier.

BERENDT J.-E. 1988. Die Welt ist Klang: Nada Brahma, Network-Medien-Cooperative, Frankfurt/M.

BERENDT J.-E. 1988. Vom Hören der Welt : Das Ohr ist der Weg, Network-MedienCooperative, Frankfurt/M.

BERTHOZ A. 2009. La simplexité. Paris: Odile Jacob.

BoHAS G. 2006. The organization of the lexicon in Arabic and other semitic languages. In Perspectives on Arabic Linguistics XVI, Papers from the Sixteenth Annual Symposium on Arabic Linguistics, Cambridge, March, 2002, ed. S. Boudelaa, 1-37. Philadelphia: John Benjamins.

BotTineAu D. 2006. «La morphosyntaxe allocutive du sens grammatical», Revue de Sémantique et Pragmatique 19/20, juin-décembre 2006, 93-120.

BotTineau D. 2007. "Ergativité nominale et actance verbale en basque », Psychomécanique $d u$ langage et linguistiques cognitives, Actes du XI Colloque International de l'AIPL, Association Internationale de Psychomécanique du Langage, Université Paul Valéry, Montpellier, 8-10 juin 2006, Lambert Lucas, 133-141.

BOTTINEAU D. 2008a. "The submorphemic conjecture in English: Towards a distributed model of the cognitive dynamics of submorphemes », Lexis 2, Toulouse.

BotTINEAU D. 2008b. "Heureux comme un poisson dans l'eau, anxieux comme un humain sous l'eau: les locutions prépositionnelles en sous, une routine énonciative variable », L'information grammaticale, 117 (mars 2008), Les locutions prépositionnelles en sous, 13-17.

BotTineAu, D. 2010a, «L'émergence du sens par l'acte de langage, de la syntaxe au submorphème », M. Banniard \& D. Philps (éds), La fabrique du signe, Linguistique de l'émergence, Toulouse: Presses Universitaires du Mirail, Interlangues, linguistique et didactique, 299-325.

BotTineau, D. 2010b, «Language and enaction », J. Stewart, O. Gapenne, E. Di Paolo (eds), Enaction: toward a new paradigm for cognitive science, MIT, 267-306.

BotTinEAU, D. 2010c, «Les linguistiques cognitives en France, problématiques actuelles », A. O. Tchubaryan, E. I. Pivovar (éds), Les sciences cognitives : questions et perspectives, Actes du $1^{\text {er }}$ séminaire franco-russe en sciences cognitives, Moscou, 21-22 septembre 2010, Académie des Sciences de Russie \& Université d'Etat pour les Sciences Humaines de Moscou, 177-222.

Bottineau, D. 2010d, «Typologie de la déflexivité », D. Bottineau, L. Begioni (éds.), La déflexivité, Langages 2010/2 (178), 89-113.

BotTineau, D. 2010e, «Les temps du verbe breton : Temps, aspect, modalité, interlocution, cognition - des faits empiriques aux orientations théoriques », C. Douay (éd.), Système et chronologie, Rennes : Presses Universitaires de Rennes, 129-157.

BotTineaU, D. 2010f. «Les locutions prépositionnelles en sur : des invariants prépositionnels aux spécialisations sémantiques », Le français moderne, 2010, 1, Danielle Leeman (dir), Locutions : continuité et innovation, 28-43.

BotTineaU, D. 2011, «La syntaxe phrastique actualise-t-elle l'incidence lexicale ? Etude comparée du français et du basque », M. Arabyan, J. Bres, D. Van Raemdonck, Th. Ponchon, R. Tremblay, P. Vachon-L'Heureux (éds.), Le Concept d'actualisation en psychomécanique, actes du XIIe Colloque international de l'Association internationale de psychomécanique du langage (AIPL), Bruxelles, 18-20 juin 2009, Limoges, LambertLucas, 103-114. 
Bottineau D. \& Roulland D. 2007a. «Le problème de la réflexivité en basque », A. Rousseau, D. Bottineau, D. Roulland (dir), L'énoncé réfléchi, Presses Universitaires de Rennes, 205-228.

BotTINEAU D. \& Roulland D. 2007b. «La grammaticalisation de l'adresse en basque : tutoiement et allocutivité », L. Begioni, C. Muller (dir.), Problèmes de sémantique et de syntaxe, Hommage à André Rousseau, Editions du Conseil Scientifique de l'Université Charles-de-Gaulle Lille 3, collection UL3 travaux et recherches, 351-372.

Bottineau D. \& Roulland D. 2010. «Les constructions impersonnelles en basque », F. Daviet-Taylor, D. Bottineau (dir.), L'impersonnel, Presses Universitaires de Rennes, 243263.

BÜHLER, K. [1934] 1990. Theory of Language, The Representational Function of Language, Translated by Donald Fraser Goodwin, Foundations of Semiotics 25, John Benjamins.

CAdiot P. \& VisetTi Y.-M. 2001. Pour une théorie des formes en sémantiques, motifs, profils, thèmes, Paris : PUF.

CADiOT P. \& VisetTi Y.-M. 2001. Pour une théorie des formes en sémantiques, motifs, profils, thèmes, Paris : PUF.

Clark A. 2008. Supersizing the Mind: Embodiment, Action and Cognitive Extension. Oxford: Oxford University Press.

Col G., Aptekman J., Girault S. \& Victorri B. (2010), «Compositionnalité gestaltiste et construction du sens par instructions dynamiques », Cognitextes 5, Numéro spécial AFLiCo 3 Grammaires en Construction(s), consulté le 01/04/2011 : http://cognitextes.revues.org/372

CORBALLIS M. 2003. From hand to mouth: The gestural origins of language. Princeton: Princeton University Press.

COURSIL J. 2000. La fonction muette du langage. Ibis Rouge Éditions. Guadeloupe: Presses Universitaires Creoles.

Cotte, P. (1996a), "Système de la syntaxe et refiguration du monde » in Cahiers de praxématique, $27,129-48$.

COWLEY S. 2007. « Cognitive dynamics and distributed language ». Language Sciences 29(5), $575-583$.

COWLEY S. 2009. «Distributed language and dynamics ». Pragmatics and Cognition 17(3), 495-507.

Cowley Stephen J., Major João C., StefFensen Sune V., Dinis A. (Eds). 2010. Signifying Bodies: Biosemiosis, Interaction and Health, The Faculty of Portuguese Catholic University.Philosophy of Braga.

CROFT b. (2009), "Toward a social cognitive linguistics", V. Evans and S. Pourcel (eds), New Directions in Cognitive Linguistics, Benjamins, 395-420.

Culioli A. [1981] 1990. «Sur le concept de notion ». BULAG $n^{\circ} 8$, repris dans Pour une linguistique de l'énonciation, t.1 : Opérations et représentations, Gap : Ophrys.

De JAegher H. \& Di PAOLO E.A. 2007. «Participatory sense-making : An enactive approach to social cognition ». Phenomenology and the Cognitive Sciences 6(4), 485-507.

DENEZ, P. (1986), «Contraintes formelles de la structuration de la phrase bretonne », in CARRER, Philippe et alii (1986), Permanence de la langue bretonne, de la linguistique à la psychanalyse, Institut Culturel de Bretagne / Skol Uhel ar Vro, Rennes, 19-44.

DiNIS A. 2010. «From my-self to our-selves : persons aseither 'individual substances' or 'relations' in Cowley Stephen J., Major João C., STEFFEnsen Sune V., Dinis A. (Eds). 2010. Signifying Bodies: Biosemiosis, Interaction and Health, The Faculty of Portuguese Catholic University.Philosophy of Braga, 75-100.

DoUAY, C. (2000), Eléments pour une théorie de l'interlocution, Un autre regard sur la grammaire anglaise, Presses Universitaires de Rennes. 
DROR I. E. \& HARNAD S. 2006. Cognition Distributed: how cognitive technology extends our minds. Amsterdam \& Philadelphia: Benjamins.

ERARD Y. 1998. "De l'énonciation à l'enaction. L'inscription corporelle de la langue », Cahiers de l'Institut de Linguistique et des Sciences du Langage, $n^{\circ} 11$, Mélanges offerts en hommage à Mortéza Mahmoudian, tome I et II.

Evans V. \& Pourcel S. 2009. New Directions in Cognitive Linguistics. Amsterdam and Philadelphia: John Benjamins.

FAUCONNIER, G. 1984. Espaces mentaux. Aspects de la construction du sens dans les langues naturelles. Paris : Editions de Minuit.

Feldman, J., AND NARAYANAN, S. 2004. "Embodied meaning in a neural theory of language". Brain and Language 89:385-392.

ForTiS, J.-M. «Sémantique cognitive et espace». Texto ! décembre 1996 [en ligne]. Disponible sur : <http://www.revue-texto.net/Inedits/Fortis_Espace.html>. (Consultée le 27/04/2011).

FRITH C. 2007. Making up the Mind. Blackwell.

FuCHS C. (dir.) 2004. La linguistique cognitive. Paris: Editions Ophrys.

GALlESE V. AND LAKOFF G. 2005. "The brain's concepts: the role of the sensory-motor system in conceptual knowledge". Cognitive Neuropsychology 22 (3/4): 455-479.

GARDINER A. H. [1932] 1951. The theory of speech and language. Oxford: Clarendon Press.

GARDNER H. 1985. The Mind's New Science. A History of the Cognitive Revolution. New York: Basic Books.

GoODY J. 1979. La raison graphique. Paris : Minuit.

GOLDBERG A. 1995. Constructions: A construction grammar approach to argument structure, Chicago : University of Chicago Press.

GoldBerg A. E. 2006. Constructions at Work. The Nature of Generalization in Language, Oxford : Oxford University Press.

Guillaume G. 1964. Langage et science du langage, Paris \& Québec : Nizet \& Presses de l'Université Laval.

Guillaume G. 1971-1999. Leçons de linguistique, 18 vol., Québec: Les Presses de l'Université Laval; Lille : Presses Universitaires de Lille.

Guillaume G. 2003. Prolégomènes à la linguistique structurale 1. Québec: Les Presses de l'Université Laval.

Guillaume G. 2004. Prolégomènes à la linguistique structurale 2. Québec: Les Presses de l'Université Laval.

Havelange V., Lenay C., AND Stewart J. 2003. "Les représentations: Mémoire externe et objets techniques ». Intellectica 35:115-131.

HOFFMEYER J. 2008. Biosemiotics : An examination into the Signs of Life and the Life of Signs. Scranton and London: University of Scranton Press.

JАСОВ A. 1970. Les exigences théoriques de la linguistique de la linguistique selon Gustave Guillaume. Paris : Klincksieck.

JAKOBSON R. 1963. Essais de linguistique générale. Tome I: les fondations du langage. Paris : Editions de Minuit, 1963.

JANET P. [1889] 2005. L'automatisme psychologique - Essai de psychologie expérimentale sur les formes inférieures de l'activité humaine. Paris : L'Harmattan.

JoHnson M. 1987. The Body in the Mind: The Bodily Basis of Meaning, Imagination, and Reason. University of Chicago Press.

Jousse M. 2008. L'anthropologie du geste. Paris : Gallimard, TEL.

KELLER P.-H. 2006. Le dialogue du corps et de l'esprit. Odile Jacob, Paris.

KÖHLER W. 1929. Gestalt Psychology. New York, Liveright. Trad. française: Psychologie de la forme, Gallimard, [1964] 2000. 
Kravchenko A. V. 2004. "Essential properties of language from the point of view of autopoiesis". http://cogprints.org/4008/01/PropertiesOfLanguage.pdf.

LADMIRAL J.-R. \& LIPIANSKY E.-M. 1989. La Communication interculturelle. Paris : Armand Colin.

LAFONT R. 1978. Le travail et la langue. Paris : Flammarion.

LAKoff G. 1987. Women, Fire and Dangerous Things, What Categories Reveal about the Mind, Chicago : University of Chicago Press.

LAKOFF G. \& Johnson M. 1980. Metaphors We Live By. Chicago: Chicago University Press.

LAKOFF G. \& JOHNSON M. 1999. Philosophy In The Flesh: the Embodied Mind and its Challenge to Western Thought. Basic Books.

LANGACKER R.W. 1987. Foundations of cognitive grammar. Stanford: Stanford University Press.

LAPLANE D. 2000. La pensée d'outre-mots, la pensée sans langage et la relation penséelangage, Institut d'édition sanofi-synthelabo.

LAVIE, R.-J. 2008. «Interspeaker variation and learnability in an exemplar-based productive model », in G. Desgulier, J.-B. Guinard \& J.-R. Lapaire (éds), Du fait grammatical au fait cognitif. From Gram to Mind (2 volumes), Bordeaux: Presses Universitaires de Bordeaux.

LEROI-GOURHAN A. (1964). Le geste et la parole. I. Technique et langage. II. La mémoire et les rythmes. Paris : Albin Michel.

LE NY, J.-F. 2005. Comment l'esprit produit du sens. O. Jacob.

MCGuRK, H., \& MCDONALD, J. (1976). "Hearing lips and seeing voices". Nature, 264, 23-30.

MacNeilage P.F. 1998. "The Frame/Content theory of evolution of speech production". Behavioral and Brain Sciences, 21, 499-546.

MARgulies A. 1985. "On listening to a dream: The sensory dimensions". Psychiatry, 48, 371-381.

MCNeILl D. 2005. Gesture and thought. Chicago: University of Chicago Press.

MaturanA, H. (1978), "Biology of language: The epistemology of reality". In G. MiLler and E. LENNEBERG (eds.), Psychology and Biology of Language and Thought: Essays in Honor of Eric Lenneberg, New York: Academic Press, 27-64.

MATURANA H.R. \& VARELA F.J. 1980. Autopoiesis and cognition: the realization of the living. Reidel, Dordrecht.

Merleau-Ponty, M. 1954. Phénoménologie de la perception. Paris : Gallimard.

NEWMEYER F. J. 2003. Grammar is grammar and usage is usage. Language, 79(4), 682-707.

OGIEN A. 2007. Les formes sociales de la pensée, La sociologie après Wittgenstein. Paris : Colin.

OGIEN A. 2007. Les formes sociales de la pensée, La sociologie après Wittgenstein. Paris : Colin.

OliVIER G. \& JUAN DE MENDOZA J.L. 2001. "Generation of oculomotor images during tasks requiring visual recognition of polygons". Perceptual and Motor Skills, 92, 1233-1247.

PESCHARD I. 2004. La réalité sans représentation. La théorie enactive de la cognition et sa légitimité épistémologique. Doctorat de philosophie des sciences. Ecole Polytechnique.

PIAGET J. 1972. Le langage et la pensée chez l'enfant. Neuchâtel: Delachaux et Niestlé.

PHILPS D. 2009. "Conceptual transfer and the emergence of the sign », CogniTextes [En ligne], Volume 2। 2009, mis en ligne le 10 décembre 2009, Consulté le 18 octobre 2010. URL : http://cognitextes.revues.org/180

PHILPS D. 2010. «Stratégies de nomination du corps et émergence du signe linguistique ». In M. BANNIARD \& D. PhilPs (éds), La fabrique du signe, Linguistique de l'émergence, Toulouse : Presses Universitaires du Mirail, Interlangues, linguistique et didactique, 281298. 
Poizat M. 1996. La voix sourde, la société face à la surdité. Paris : Métailié.

PotTIER, B. 1992. Sémantique générale. Paris : PUF.

RASTIER F. 1993. "La sémantique cognitive : éléments d'histoire et d'épistémologie ». Histoire Epistémologie Langage, 15:1, 153-187.

RASTIER R. 2006. De l'origine du langage à l'émergence du milieu sémiotique. Marges linguistiques, 11.

Rizzolatti G. \& CRAighero L. 2004. "The mirror-neuron system”. Annual Review of Neuroscience, 27, 169-192.

Robert, S. (éd.). 2003. Perspectives synchroniques sur la grammaticalisation. LouvainParis : Peeters.

RosenFIELD I. 1997. L'invention de la mémoire. Paris: Champs Flammarion.

SADOWSKI P. 2001. "The sound as an echo to the sense: the iconicity of English gl- words". In O. FISCHER \& M. NÄNNY (eds), The motivated sign, iconicity in language and literature 2, Benjamins, 69-88.

SHANON B. 1993. The representational and the presentational: An essay on cognition and the study of mind. London: Harvester-Wheatsheaf.

SimONDON, G. 1989. L'individuation psychique et collective. Paris : Aubier.

SimONDON, G. 1995. L'individu et sa genèse physico-biologique. Paris : Jérôme Millon.

STEFFENSEN S. V. 2009. "Language, Languaging and the Extended Mind Hypothesis.» Pragmatics and Cognition 17(3), 677-697.

STIEGLER B. 1994. La Technique et le temps 1 : La faute d'Epiméthée. Paris : Galilée.

STIEGLER B. 2004. Philosopher par accident : Entretiens avec Elie During. Galilée.

TALMY L. 2000. Toward a Cognitive Semantics, volumes I and II. MIT Press.

ToBIN Y. 1997. Phonology as human behavior: Theoretical implications and clinical applications. Durham, NC: Duke University Press.

UEXKÜLL J. von [1934] 2004. Mondes animaux et monde humain. Pocket.

VALETTE M. 2001. «Le langage comme système complexe et auto-organisé chez Guillaume. Éléments de lecture », in P. De CARVAlHo, N. QuaYle, L. Rosier \& O. Soutet, CIPL8 / La psychomécanique aujourd'hui, Actes $d u 8^{e}$ Colloque international de psychomécanique du langage. Seyssel, 1997. Paris : Champion, 536-544.

VALETTE M. 2003. «Énonciation et cognition : deux termes in absentia pour des notions omniprésentes dans l'œuvre de Guillaume », Le français moderne, Tome LXXI, 1, 6-25.

Valette M. 2004. "Conceptualisation and Evolution of Concepts. The example of French Linguist Gustave Guillaume », in K. Fløttum \& F. Rastier, (eds.), Academic discourse multidisciplinary approaches, Oslo: Novus, 55-74.

VALETTE M. 2006. Linguistiques énonciatives et cognitives françaises. Gustave Guillaume, Bernard Pottier, Maurice Toussaint, Antoine Culioli, collection «Bibliothèque de Grammaire et de Linguistique », Paris : Honoré Champion.

VALIN R. 1981. Perspectives psychomécaniques sur la syntaxe, Laval: Les Presses de l'université Laval, Québec.

VANDELOISE C. 1986, L'espace en français, Seuil.

VARela F., Thompson E. \& Rosch E. 1993. The Embodied Mind: Cognitive Science and Human Experience. MIT Press, Cambridge.

VICTORRI, B. 1999. « Le sens grammatical », Langages, 136, 85-105.

VyGOTSKY L.S. (1962). Thought and Language. E. KAUFMANN \& G. VAKAR, eds \& trans., Cambridge: MIT Press.

VinAY J.-P. \& DARBELnet J. 1958. Stylistique comparée du français et de l'anglais. Paris : Didier.

WAWRZYNIAK J. K. 2010. Native speakers, mother tongues and natural semantic metalanguages. Language Sciences 32, 648-670. 
WHITAKER R. Encyclopaedia Autopoietica, http://www.enolagaia.com/EAIntro.html

WILSON M. 2002. "Six views of embodied cognition". Psychological Bulletin and Review, 9(4), 625-636.

ZIEMKE T. 2003. "What's that thing called embodiment?" In: Proceedings of the 25th Annual Meeting of the Cognitive Science Society. Lawrence Erlbaum. 